Publ. Mat. 46 (2002), 405-434

\title{
RESTRICTION AND DECAY FOR FLAT HYPERSURFACES
}

\author{
Anthony Carbery and Sarah Ziesler
}

\begin{abstract}
In the first part we consider restriction theorems for hypersurfaces $\Gamma$ in $\mathbf{R}^{n}$, with the affine curvature $K_{\Gamma}^{1 /(n+1)}$ introduced as a mitigating factor. Sjölin, [19], showed that there is a universal restriction theorem for all convex curves in $\mathbf{R}^{2}$. We show that in dimensions greater than two there is no analogous universal restriction theorem for hypersurfaces with non-negative curvature.

In the second part we discuss decay estimates for the Fourier transform of the density $K_{\Gamma}^{1 / 2}$ supported on the surface and investigate the relationship between restriction and decay in this setting. It is well-known that restriction theorems follow from appropriate decay estimates; one would like to know whether restriction and decay are, in fact, equivalent. We show that this is not the case in two dimensions. We also go some way towards a classification of those curves/surfaces for which decay holds by giving some sufficient conditions and some necessary conditions for decay.
\end{abstract}

\section{Universal restriction theorems}

If $S$ is a smooth ( $n-1)$-dimensional submanifold in $\mathbf{R}^{n}(n \geq 3)$, $S_{0}$ is a compact subset with non-vanishing Gaussian curvature and $d \sigma$ is the induced Lebesgue measure then the Stein-Tomas restriction theorem says that, for all $f \in L^{p}\left(\mathbf{R}^{n}\right)$,

$$
\left(\int_{S_{0}}|\hat{f}(\xi)|^{q} d \sigma(\xi)\right)^{1 / q} \leq C\|f\|_{p},
$$

2000 Mathematics Subject Classification. 42B99.

Key words. Restriction, decay estimates, affine curvature.

The authors were partially supported by Forbairt.

The first author was partially supported by a Leverhulme Fellowship.

The second author would like to thank Carlos E. Kenig for many valuable conversations relating to this work. 
for $1 \leq p \leq \frac{2 n+2}{n+3}, q \leq\left(\frac{n-1}{n+1}\right) p^{\prime}$, where $\frac{1}{p}+\frac{1}{p^{\prime}}=1$. (See $[\mathbf{2 2}]$, and [25] in the case of the sphere.) The inequality (1) is known as the $L(p, q)$ restriction property. Here and throughout, $C$ denotes a universal constant whose value may change from line to line. It is also well-known that (1) must fail for $p \geq \frac{2 n}{n+1}$ and for $q>\left(\frac{n-1}{n+1}\right) p^{\prime}$; see [22] again. However, for $n=2$ (1) is known for the larger and optimal range, $1 \leq$ $p<4 / 3, q \leq p^{\prime} / 3$; see $[\mathbf{2 8}]$ and $[\mathbf{2 2}]$.

There are various related results for surfaces whose Gaussian curvature may vanish but which nevertheless satisfy some other conditions such as being finite-type or having non-vanishing principal curvatures; see for example $[\mathbf{2 2}],[\mathbf{9}],[\mathbf{1 8}],[\mathbf{2 0}]$.

In higher dimensions, in the case of non-vanishing Gaussian curvature, some exciting progress has been made in extending the range of $p$; see $[\mathbf{3}],[\mathbf{2}],[\mathbf{2 7}],[\mathbf{1 6}],[\mathbf{1 7}],[\mathbf{2 3}]$ and for the current best result in three dimensions see $[\mathbf{2 4}]$.

In this paper we consider hypersurfaces which may be flat. We take surfaces $\Gamma(t)=(t, \gamma(t))$ where $t \in \mathbf{R}^{n-1}$ and $\gamma: \mathbf{R}^{n-1} \longrightarrow \mathbf{R}$. We begin by formulating the appropriate analogue of (1) for such surfaces. In our discussion of restriction theorems for such surfaces in the present paper, when $n \geq 3$, we only consider the range of $p, q$ in the SteinTomas theorem and do not attempt an analogue of the improved higherdimensional results. To compensate for the possible lack of curvature we insert a mitigating factor (which we shall choose in a moment) $M_{\Gamma}(t)$ into the left-hand-side of (1) and look for inequalities of the form

$$
\left(\int|\hat{f}(\Gamma(t))|^{q} \psi(t) M_{\Gamma}(t) d t\right)^{1 / q} \leq C\|f\|_{p} .
$$

Here, to obtain positive results, we shall see that it is appropriate to take $\psi$ to be a smooth non-negative cut-off function when $n \geq 4$ but in dimensions 2 and 3 we can take $\psi$ to be the characteristic function of the set where $\Gamma$ is defined.

One alternative approach in the absence of curvature, followed by Bak, $[\mathbf{1}]$, is to look for Orlicz space theorems. Another approach, taken by Brandolini, Iosevich and Travaglini, [4], is to average over rotations.

We would like to choose $M_{\Gamma}$ to reflect the affine-invariance of the restriction property. Following Sjőlin, [19], and also [7], when $q=$ $\left(\frac{n-1}{n+1}\right) p^{\prime}$ it is natural to take $M_{\Gamma}$ to be the affine curvature $K_{\Gamma}(t)^{1 /(n+1)}$ where $K_{\Gamma}(t)=\operatorname{det}(\operatorname{Hess} \gamma(t))$. The power $\frac{1}{n+1}$ is the unique power of $K_{\Gamma}$ which preserves the affine invariance of the problem; the resulting 
inequality is then also invariant under reparametrisation of the hypersurface and suggests that the restriction phenomenon might be a "universal" or intrinsic property of a class of hypersurfaces.

This power of $K_{\Gamma}$ preserves affine invariance when $q=\left(\frac{n-1}{n+1}\right) p^{\prime}$. However in general we need $K_{\Gamma}^{\frac{q}{p^{\prime}(n-1)}}$ in order to have affine invariance in an $L(p, q)$ restriction theorem. Note that $p=1$ gives the power 0 .

So we wish to determine for which $\Gamma$ one has

$$
\left(\int|\hat{f}(\Gamma(t))|^{q} \psi(t) K_{\Gamma}(t)^{\frac{q}{p^{\prime}(n-1)}} d t\right)^{1 / q} \leq C\|f\|_{p}
$$

for $1 \leq p \leq \frac{2 n+2}{n+3}, q \leq\left(\frac{n-1}{n+1}\right) p^{\prime}$. If one can show that

$$
\left(\int|\hat{f}(\Gamma(t))|^{2} \psi(t) K_{\Gamma}(t)^{\frac{1}{n+1}} d t\right)^{1 / 2} \leq C\|f\|_{p}
$$

for $p=\frac{2 n+2}{n+3}$ then interpolation with the $p=1$ case gives the full range. To distinguish the cases where $\psi$ is a smooth cut-off or a characteristic function we shall refer to the above inequality as $(2)^{*}$ when we mean to take $\psi$ as a characteristic function.

In two dimensions Sjolin, [19], proved that there is a universal restriction theorem for all convex curves:

Theorem $1.1([\mathbf{1 9}])$. Let $\Gamma(t)=(t, \gamma(t))$, where $\gamma \in C^{2}(0,1)$ is convex.

Then (2)* holds for $1 \leq p<\frac{4}{3}, q=\frac{1}{3} p^{\prime}$ and with a constant $C$ independent of $\gamma$.

It is worth noting that the proof of this given in [19] also applies in the following case.

Theorem 1.2. Let $\Gamma(t)=(t, \gamma(t))$, where $\gamma: \mathbf{R} \longrightarrow \mathbf{R}$, and $\gamma \in C^{2}$ is convex.

Then $\left(\int|\hat{f}(t, \gamma(t))|^{q} \gamma^{\prime \prime}(t)^{q / p^{\prime}} d t\right)^{1 / q} \leq C\|f\|_{p}$ holds for $1 \leq p<\frac{4}{3}$, $q=\frac{1}{3} p^{\prime}$ and with a constant $C$ independent of $\gamma$.

We also note that the proof only requires that $\gamma^{\prime \prime}$ be single-signed and the argument will go through in exactly the same manner if one assumes that $\gamma$ is concave instead of convex. 
We now consider the situation in higher dimensions. We shall demonstrate that there is no universal restriction theorem of the form $(2)^{*}$ for hypersurfaces whose curvature is non-negative, in dimensions $n \geq 3$. We let $t=\left(t_{1}, t_{2}, \ldots, t_{n-1}\right)$ and consider surfaces of the form $\Gamma(t)=(t, \gamma(t))$, where $\gamma: \mathbf{R}^{n-1} \longrightarrow \mathbf{R}$ is $C^{2}[(0,1) \times(0,1) \times \cdots \times(0,1)]$ and satisfies $\operatorname{det}(\operatorname{Hess} \gamma(t)) \geq 0$.

Theorem 1.3. Let $\Gamma$ be as described above and $p>1$. Then there is no universal constant $C$ such that $(2)^{*}$ holds for all such $\Gamma$.

Proof: Let $t_{1}=r \cos \theta, t_{2}=r \sin \theta, y=\left(t_{3}, \ldots, t_{n-1}\right)$ and define $\gamma(t)=r \cos N \theta+|y|^{2}$. Then $K_{\Gamma}(t)=2^{n-3} \frac{N^{2} \sin ^{2} N \theta}{r^{2}} \geq 0$. We now choose $\hat{f}\left(\xi_{1}, \xi_{2}, \ldots, \xi_{n}\right)=\phi_{1}\left(\xi_{1}, \xi_{2}, \ldots, \xi_{n-1}\right) \phi_{2}\left(\xi_{n}\right)$ where $\phi_{1}$ and $\phi_{2}$ are smooth non-negative cut-off functions such that $\left|\phi_{1}\right| \leq 1,\left|\phi_{2}\right| \leq 1$,

$$
\phi_{1}\left(\xi_{1}, \ldots, \xi_{n-1}\right) \equiv \begin{cases}1 & \text { for }\left|\left(\xi_{1}, \ldots, \xi_{n-1}\right)\right| \leq \frac{1}{2} \\ 0 & \text { for }\left|\left(\xi_{1}, \ldots, \xi_{n-1}\right)\right| \geq 1\end{cases}
$$

and

$$
\phi_{2}\left(\xi_{n}\right) \equiv \begin{cases}1 & \text { for }\left|\xi_{n}\right| \leq 2 \\ 0 & \text { for }\left|\xi_{n}\right| \geq 4\end{cases}
$$

Then $(2)^{*}$ gives

$$
\iiint\left|\phi_{1}(r, \theta, y)\right|^{q}\left|\phi_{2}(\tilde{\gamma}(r, \theta, y))\right|^{q}\left\{\frac{N^{2} \sin ^{2} N \theta}{r^{2}}\right\}^{\frac{q}{p^{\prime}(n-1)}} r d r d \theta d y \leq C,
$$

where $\tilde{\gamma}$ is $\gamma$ written in cylindrical polar coordinates. Using the fact that $|\gamma| \leq 2$ on the support of $\phi_{1}$ this leads to

$$
\int_{0}^{\pi / 2}(N \sin N \theta)^{\frac{2 q}{p^{\prime}(n-1)}} d \theta \leq C .
$$

Since $\int_{0}^{\pi / 2}(\sin N \theta)^{\frac{2 q}{p^{\prime}(n-1)}} d \theta \geq c,(3)$ must fail as $N \longrightarrow \infty$.

In the setting of convex hypersurfaces the question seems considerably more subtle and we have not arrived at any definitive conclusion. The standard method to prove restriction results is to use decay estimates for the Fourier transform of the measure $K_{\Gamma}^{\frac{1}{2}}$ supported on the surface and these estimates may fail even for finite-type convex surfaces, sometimes quite dramatically, as was shown in [6]. By finite-type we mean that the surface has finite order of contact with any tangent line, as defined in [5]. We shall discuss decay estimates further, in the context of radial hypersurfaces, in Section 4 and Section 5. The standard means of 
finding a counterexample to restriction (via a Knapp-type homogeneity argument, see $[\mathbf{2 2}],[\mathbf{1 0}],[\mathbf{1 1}]$ ) leads one to an inequality which is true for all convex hypersurfaces, as we shall see below. This leads one to wonder whether there could indeed be a universal restriction theorem for convex hypersurfaces in $\mathbf{R}^{n}, n \geq 3$.

For convex hypersurfaces with $\gamma \geq 0$ the natural approach when looking for a counterexample is to take a smooth non-negative cut-off function $\phi$ which is identically 1 on $(0,1)$ and define $\hat{f}\left(\xi_{1}, \xi_{2}, \ldots, \xi_{n}\right)=$ $\phi\left(\frac{\xi_{1}}{\delta_{1}}\right) \phi\left(\frac{\xi_{2}}{\delta_{2}}\right) \cdots \phi\left(\frac{\xi_{n-1}}{\delta_{n-1}}\right) \phi\left(\frac{\xi_{n}}{\gamma\left(\delta_{1}, \delta_{2}, \ldots, \delta_{n-1}\right)}\right)$. Then if $(2)^{*}$ holds for $\Gamma$ we have, for $0<\delta_{i} \leq 1, i=1,2, \ldots, n-1$,

$\int_{0}^{\delta_{n-1}} \cdots \int_{0}^{\delta_{1}} K_{\Gamma}(t)^{\frac{q}{p^{\prime}(n-1)}} d t_{1} \cdots d t_{n-1} \leq C\left[\delta_{1} \cdots \delta_{n-1} \gamma\left(\delta_{1}, \ldots, \delta_{n-1}\right)\right]^{q / p^{\prime}}$

In fact, one can easily check that the optimal choice of the parameters is $\delta_{1}=\delta_{2}=\cdots=\delta_{n-1}=1$. Then the above inequality becomes

$$
\int_{0}^{1} \cdots \int_{0}^{1} K_{\Gamma}^{\frac{q}{p^{\prime}(n-1)}} d t_{1} \cdots d t_{n-1} \leq C \gamma(1, \ldots, 1)^{q / p^{\prime}}
$$

However the isoperimetric inequality of affine differential geometry (see for example [13, Chapter 5] and [14]) shows that (4) holds. A simple argument, which we now give for the sake of completeness, verifies this in the case of radial hypersurfaces. We let $\Gamma(t)=(t, \gamma(|t|))$ in $\mathbf{R}^{n}$, where $n \geq 3$. In this case, $K_{\Gamma}(t)$ is given by $K_{\Gamma}(t)=\gamma^{\prime \prime}(|t|)\left(\frac{\gamma^{\prime}(|t|)}{|t|}\right)^{n-2}$. Clearly, if $\gamma$ is convex and $\gamma^{\prime}(0)=0$ then $K_{\Gamma} \geq 0$ for $\gamma \in C^{2}(0,1)$ and moreover $\Gamma$ is convex.

Then (4) can be modified to

$$
\int_{0}^{1} K_{\Gamma}(r)^{\frac{q}{p^{\prime}(n-1)}} r^{n-2} d r \leq C \gamma(1)^{q / p^{\prime}}
$$

Lemma 1.1. When $p^{\prime} \geq\left(\frac{n+1}{n-1}\right) q$, (5) holds uniformly for all convex $\gamma \in C^{2}(0,1)$ such that $\gamma(0)=\gamma^{\prime}(0)=0$. 
Proof: We use $\int_{0}^{1} \gamma^{\prime \prime}(r)(1-r) d r=\gamma(1)$ and Hőlder's inequality to obtain

$$
\begin{aligned}
& \left(\int_{0}^{1} \gamma^{\prime \prime}(r)^{\frac{q}{p^{\prime}(n-1)}} \gamma^{\prime}(r)^{\frac{q(n-2)}{p^{\prime}(n-1)}} r^{(n-2)\left(1-\frac{q}{p^{\prime}(n-1)}\right)} d r\right) \\
& \quad=\int_{0}^{1}\left[\gamma^{\prime \prime}(r)(1-r)\right]^{\frac{q}{p^{\prime}(n-1)}} \frac{\gamma^{\prime}(r)^{\frac{q(n-2)}{p^{\prime}(n-1)}}}{(1-r)^{\frac{q}{p^{\prime}(n-1)}}} r^{\frac{(n-2)\left(p^{\prime}(n-1)-q\right)}{p^{\prime}(n-1)}} d r \\
& \quad \leq \gamma(1)^{\frac{q}{p^{\prime}(n-1)}}\left(\int_{0}^{1} \frac{\gamma^{\prime}(r)^{\frac{q(n-2)}{p^{\prime}(n-1)-q}}}{(1-r)^{\frac{q}{p^{\prime}(n-1)-q}}} r^{n-2} d r\right)^{\frac{p^{\prime}(n-1)-q}{p^{\prime}(n-1)}} \\
& \quad \leq \gamma(1)^{\frac{q}{p^{\prime}(n-1)}}\left(\int_{0}^{1} \gamma^{\prime}(r) d r\right)^{\frac{q(n-2)}{p^{\prime}(n-1)}}\left(\int_{0}^{1} \frac{r^{\frac{\left.(n-2)(n-1) p^{\prime}-q\right]}{(n-1)\left(p^{\prime}-q\right)}}}{(1-r)^{\frac{q}{(n-1)\left(p^{\prime}-q\right)}}} d r\right)^{\frac{p^{\prime}-q}{p^{\prime}}} \\
& \leq C \gamma(1)^{\frac{q}{p^{\prime}}}\left(\int_{0}^{1} \frac{1}{(1-r)^{\frac{q}{(n-1)\left(p^{\prime}-q\right)}}} d r\right)^{\frac{p^{\prime}-q}{p^{\prime}}} \\
& \leq C \gamma(1)^{\frac{q}{p^{\prime}}},
\end{aligned}
$$

when $\frac{p^{\prime}}{q} \geq \frac{n+1}{n-1}$.

\section{Restriction and decay}

With the exception of the argument for $n=2$, the typical proof of an $L(p, 2)$ restriction theorem hinges on a decay estimate such as

$$
\left|\int_{S} e^{i \xi \cdot x} d \sigma(x)\right| \leq \frac{C}{(1+|\xi|)^{r}},
$$

where $r>0$ and $d \sigma$ is the surface measure on $S$. If (6) holds then one obtains an $L(p, 2)$ restriction theorem for the surface $S$, for $p \leq \frac{2(r+1)}{r+2}$. The key value of $r$ is $r=\frac{n-1}{2}$. See for example, [22].

Some recent work of Iosevich, [10], and Iosevich and $\mathrm{Lu},[\mathbf{1 1}]$, shows that in the cases of hypersurfaces with non-vanishing Gaussian curvature or convex finite-type hypersurfaces, restriction and decay (both without mitigating factors) are equivalent. More precisely, (2) holds for $p=$ $\frac{2(r+1)}{r+2}, q=2$ if, and only if, (6) holds. 
In our setting, the appropriate decay estimate to give restriction is given in the following lemma. Part b) of the lemma also shows that if the decay estimate misses by a logarithm, then one obtains restriction up to the endpoint. The proof is an easy adaptation of the arguments in $[\mathbf{1 2}]$; see also $[\mathbf{8}]$ where the ideas originated.

Lemma 2.1. Suppose that $\Gamma$ is defined on the open set $\Omega \subset \mathbf{R}^{n-1}$, $\Gamma: \Omega \longrightarrow \mathbf{R}^{n}$ and $\Gamma$ is $C^{2}(\Omega)$.

a) If, for $0 \leq \beta \leq \frac{1}{2}$, and some $N>0$,

$$
\left.\left|\int_{\Omega} e^{i(\xi \cdot \Gamma(t))}\right| K_{\Gamma}(t)\right|^{\beta+i \alpha} d t \mid \leq C \frac{(1+|\alpha|)^{N}}{\left|\xi_{n}\right|^{\beta(n-1)}},
$$

then $(2)^{*}$ holds for $p=\frac{2(1+(n-1) \beta)}{2+(n-1) \beta}, q=2$.

b) If, for $0 \leq \beta \leq \frac{1}{2}$, some $N>0$ and some $\epsilon>0$,

$$
\left.\left|\int_{\Omega} e^{i(\xi \cdot \Gamma(t))}\right| K_{\Gamma}(t)\right|^{\beta+i \alpha} d t \mid \leq C \frac{(1+|\alpha|)^{N} \log ^{\epsilon}\left|\xi_{n}\right|}{\left|\xi_{n}\right|^{\beta(n-1)}},
$$

then $(2)^{*}$ holds for $p<\frac{2(1+(n-1) \beta)}{2+(n-1) \beta}, q=2$.

A corresponding statement holds for (2) if one inserts an appropriate cut-off into the integral. This will be needed when we discuss dimensions $n \geq 4$.

If $\beta=\frac{1}{2}$ in Lemma 2.1a) then one has $p=\frac{2 n+2}{n+3}$ and so interpolation gives the full result, i.e. (2) for $1 \leq p \leq \frac{2 n+2}{n+3}, q \leq\left(\frac{n-1}{n+1}\right) p^{\prime}$. If $\beta=\frac{1}{2}$ in b) then we obtain (2) for $1 \leq p<\frac{2 n+2}{n+3}, q<\left(\frac{n-1}{n+1}\right) p^{\prime}$.

Decay estimates with mitigating factors have previously been considered in $[\mathbf{1 2}],[\mathbf{2 1}],[\mathbf{6}]$ and $[\mathbf{1 8}]$.

In Section 3 we shall show that, in our setting, restriction and decay are not equivalent when $n=2$. There are in fact convex curves for which the decay estimate fails.

In Section 3 and Section 4 we also give a partial classification of the curves/surfaces for which the desired decay estimates hold, in $\mathbf{R}^{2}$ and $\mathbf{R}^{3}$ respectively. The higher-dimensional case is considered in Section 5. In dimensions three and above we consider only radial hypersurfaces $\Gamma(t)=$ $(t, \gamma(|t|))$.

Before proceeding, we state Van der Corput's lemma, a crucial tool in our estimates. See $[\mathbf{2 2}]$ for the proof. 
Lemma $2.2([\mathbf{2 2}])$. Suppose that $\phi$ is real-valued and smooth on $[a, b]$ and $\psi$ is real-valued and $C^{1}([a, b])$. If $\left|\phi^{(k)}(x)\right| \geq 1$, then

$$
\left|\int_{a}^{b} e^{i \lambda \phi(x)} \psi(x) d x\right| \leq c_{k} \lambda^{-1 / k}\left[|\psi(b)|+\int_{a}^{b}\left|\psi^{\prime}(x)\right| d x\right],
$$

when i) $k \geq 2$, ii) $k=1$, if $\phi^{\prime}$ is monotonic.

It follows that if $\psi^{\prime}$ is single-signed then one can replace the righthand-side of the estimate by $c_{k} \lambda^{-1 / k} \max _{[a, b]}|\psi(x)|$; we shall use this repeatedly.

\section{Restriction and decay in 2-dimensions}

Theorem 3.1. There is a $\gamma:[0,1] \longrightarrow \mathbf{R}$ such that $\gamma \in C^{2}(0,1), \gamma(0)=$ $\gamma^{\prime}(0)=0, \gamma$ is convex and

$$
\left|\int_{0}^{1} e^{i \xi \cdot(t, \gamma(t))} \gamma^{\prime \prime}(t)^{1 / 2} d t\right| \leq \frac{C_{\gamma}}{\left|\xi_{2}\right|^{1 / 2}}
$$

fails.

It follows from this, together with Theorem 1.1, that restriction and decay are not equivalent in a general setting. To prove the theorem we first note that, taking $\xi_{1}=0$ in the decay estimate one obtains

$$
\left|\int_{0}^{1} e^{i \xi_{2} \cdot \gamma(t)} \gamma^{\prime \prime}(t)^{1 / 2} d t\right| \leq \frac{C_{\gamma}}{\left|\xi_{2}\right|^{1 / 2}}
$$

and so if we choose $\xi_{2}$ so that $\xi_{2} \gamma(1) \approx \frac{1}{100}$ then

$$
\int_{0}^{1} \gamma^{\prime \prime}(t)^{1 / 2} d t \leq \frac{C_{\gamma}}{\left|\xi_{2}\right|^{1 / 2}} \leq C_{\gamma} \gamma(1)^{1 / 2}
$$

Proof of Theorem 3.1: By (8) it is enough to show that there is a $\gamma:[0,1] \longrightarrow R$ so that $\gamma(1)$ is finite but $\int_{0}^{1} \gamma^{\prime \prime}(t)^{1 / 2} d t$ is not. For example, we can choose $\gamma$ so that

$$
\gamma^{\prime \prime}(t)=\frac{1}{(1-t)^{2} \log ^{2} \frac{1}{1-t}},
$$

for $1 / 2 \leq t \leq 1$. Then $\gamma^{\prime \prime}(t)^{1 / 2}$ is not integrable at 1 . However, for $1 / 2 \leq t<1$,

$$
\gamma^{\prime}(t)=\gamma^{\prime}(1 / 2)+\int_{1 / 2}^{t} \gamma^{\prime \prime}(s) d s
$$


and

$$
\gamma(t)=\gamma(1 / 2)+(t-1 / 2) \gamma^{\prime}(1 / 2)+\int_{1 / 2}^{t}(t-s) \gamma^{\prime \prime}(s) d s,
$$

for $1 / 2 \leq t \leq 1$, so that

$$
\gamma(1)=\gamma(1 / 2)+1 / 2 \gamma^{\prime}(1 / 2)+\int_{1 / 2}^{1} \frac{1}{(1-s) \log ^{2} \frac{1}{1-s}} d s,
$$

which is finite.

This shows that the $L(6 / 5,2)$ restriction theorem for convex curves does not imply the corresponding decay estimate with mitigating factor $K_{\Gamma}^{1 / 2}$. We do not know whether restriction and decay are equivalent for $\beta<1 / 2$. In this case, the inequality analogous to (8) is $\int_{0}^{1} \gamma^{\prime \prime}(t)^{\beta} d t \leq$ $C_{\gamma} \gamma(1)^{\beta}$, which is true for all convex curves with $\gamma(0)=\gamma^{\prime}(0)=0$.

We now consider necessary/sufficient conditions for uniform decay estimates. Some results with a similar flavour can be found in [15].

Preliminary Remark. Let us suppose for a moment that we have uniform decay for the class of curves $\gamma$ that are $C^{2}(0,1)$ and convex. (So the decay estimate (7) holds with a constant independent of $\gamma$.) Then the argument used for (8) together with dilation shows that

$$
\int_{0}^{\delta} \gamma^{\prime \prime}(t)^{1 / 2} d t \leq C \gamma(\delta)^{1 / 2}
$$

for all $\delta \leq 1$, with $C$ independent of $\gamma$.

Proposition 3.1. Suppose that $\gamma:[0,1] \longrightarrow R$ satisfies $\gamma, \gamma^{\prime}$ convex, $\gamma \in C^{2}(0,1]$ and $\gamma \in C^{3}(0,1), \gamma(0)=\gamma^{\prime}(0)=0$ and $\left(\frac{\gamma^{\prime \prime 1 / 2}}{\gamma^{\prime}}\right)^{\prime} \leq 0$. If also $\gamma^{\prime \prime}(t) \leq C \frac{\gamma^{\prime}()^{2}}{\gamma(t)}$, for all $0 \leq t \leq 1$, then

$$
\left|\int_{0}^{1} e^{i(\xi t+\eta \gamma(t))} \gamma^{\prime \prime}(t)^{1 / 2+i \alpha} d t\right| \leq \frac{C(1+|\alpha|)}{|\eta|^{1 / 2}} \text { for all } \eta,
$$

with $C$ independent of $\gamma$.

Conversely, if $\gamma:[0,1] \longrightarrow R$ is $C^{2}(0,1)$, convex and $\left(\frac{\gamma^{\prime \prime 1 / 2}}{\gamma^{\prime}}\right)^{\prime} \leq 0$ and (10) holds for $\gamma$ and all of its dilates $\gamma(\delta \cdot)$, for $\delta \leq 1$, then $\gamma^{\prime \prime}(t) \leq$ $C \frac{\gamma^{\prime}(t)^{2}}{\gamma(t)}$, for all $0 \leq t \leq 1$ and with $C$ independent of $\gamma$. 
Remarks. 1. The converse statement is a corollary of (9); in fact the condition $\gamma^{\prime \prime} \leq C \frac{\gamma^{\prime 2}}{\gamma}$ is equivalent to (9), under the assumption that $\left(\frac{\gamma^{\prime \prime 1 / 2}}{\gamma^{\prime}}\right)^{\prime} \leq 0$.

2. This result also shows that decay holds for flat curves such as $\gamma(t)=e^{-1 / t^{m}}$ and $\gamma(t)=\frac{t}{\log 1 / t}$, for $t$ small.

3 . We have only considered the mitigating factor with power of the curvature $\beta=1 / 2$; conditions could also be formulated for $\beta<$ $1 / 2$.

Proof: We first prove the forward assertion. We begin by noting that

$$
\begin{aligned}
\int_{0}^{\gamma^{-1}\left(\frac{1}{|\eta|}\right)} \gamma^{\prime \prime}(t)^{1 / 2} d t & \leq C \int_{0}^{\gamma^{-1}\left(\frac{1}{|\eta|}\right)} \frac{\gamma^{\prime}(t)}{\gamma(t)^{1 / 2}} d t \\
& =\frac{C}{|\eta|^{1 / 2}} .
\end{aligned}
$$

So, we need only consider $\int_{\gamma^{-1}\left(\frac{1}{|\eta|}\right)}^{1} e^{i(\xi t+\eta \gamma(t))} \gamma^{\prime \prime}(t)^{1 / 2+i \alpha} d t$. We suppose that $\xi+\eta \gamma^{\prime}\left(t_{0}\right)=0$ for $t_{0} \in\left[\gamma^{-1}\left(\frac{1}{|\eta|}\right), 1\right]$. If there is no such $t_{0}$ then the argument below simplifies. For such a $t_{0}$ to exist, clearly $\xi$ and $\eta$ must have opposite signs and so $\gamma^{\prime}\left(t_{0}\right)=\left|\frac{\xi}{\eta}\right|$. We then let $t_{1}$ and $t_{2}$ be such that $\gamma^{\prime}\left(t_{1}\right)=\frac{1}{2} \gamma^{\prime}\left(t_{0}\right)$ and $\gamma^{\prime}\left(t_{2}\right)=2 \gamma^{\prime}\left(t_{0}\right)$. We assume that $\gamma^{-1}\left(\frac{1}{|\eta|}\right)<t_{1}<t_{0}<t_{2} \leq 1$. The other cases are either similar or simpler. Then $\left|\xi+\eta \gamma^{\prime}(t)\right| \geq c|\xi|$ and $\left|\xi+\eta \gamma^{\prime}(t)\right| \geq \tilde{c}|\eta| \gamma^{\prime}(t)$ on $\left[0, t_{1}\right]$ and $\left[t_{2}, 1\right]$. Then, using Van der Corput's lemma, together with the convexity of $\gamma^{\prime}$ and the assumption $\gamma^{\prime \prime} \leq C \frac{\gamma^{\prime 2}}{\gamma}$, we have

$$
\begin{aligned}
\left|\int_{\gamma^{-1}\left(\frac{1}{|\eta|}\right)}^{t_{1}} e^{i(\xi t+\eta \gamma(t))} \gamma^{\prime \prime}(t)^{1 / 2+i \alpha} d t\right| & \leq \frac{C(1+|\alpha|)}{|\xi|} \max _{t \in\left[\gamma^{-1}\left(\frac{1}{|\eta|}\right), t_{1}\right]} \gamma^{\prime \prime}(t)^{1 / 2} \\
& \leq \frac{C(1+|\alpha|)}{|\xi|} \frac{\gamma^{\prime}\left(t_{1}\right)}{\gamma\left(t_{1}\right)^{1 / 2}} \\
& \leq \frac{C(1+|\alpha|)}{|\xi|} \frac{\gamma^{\prime}\left(t_{1}\right)}{\frac{1}{|\eta|^{1 / 2}}} \\
& =\frac{C(1+|\alpha|)}{|\eta|^{1 / 2}} .
\end{aligned}
$$


Also using Van der Corput we obtain

$$
\left|\int_{t_{1}}^{t_{2}} e^{i(\xi t+\eta \gamma(t))} \gamma^{\prime \prime}(t)^{1 / 2+i \alpha} d t\right| \leq \frac{C(1+|\alpha|)}{|\eta|^{1 / 2}} \frac{\max _{t \in\left[t_{1}, t_{2}\right]} \gamma^{\prime \prime}(t)^{1 / 2}}{\min _{t \in\left[t_{1}, t_{2}\right]} \gamma^{\prime \prime}(t)^{1 / 2}} .
$$

To see that the right-hand side is bounded by $\frac{C(1+|\alpha|)}{|\eta|^{1 / 2}}$ we need to use the assumptions that $\gamma^{\prime}$ is convex and $\frac{\gamma^{\prime \prime 1 / 2}}{\gamma^{\prime}}$ is decreasing. Then

$$
\frac{\max _{t \in\left[t_{1}, t_{2}\right]} \gamma^{\prime \prime}(t)^{1 / 2}}{\min _{t \in\left[t_{1}, t_{2}\right]} \gamma^{\prime \prime}(t)^{1 / 2}} \leq \frac{\gamma^{\prime \prime}\left(t_{2}\right)^{1 / 2}}{\gamma^{\prime \prime}\left(t_{1}\right)^{1 / 2}} \leq \frac{\gamma^{\prime}\left(t_{2}\right)}{\gamma^{\prime}\left(t_{1}\right)}=4
$$

For the remaining part of the integral we integrate by parts.

$$
\begin{aligned}
& \left|\int_{t_{2}}^{1} e^{i(\xi t+\eta \gamma(t))} \gamma^{\prime \prime}(t)^{1 / 2+i \alpha} d t\right| \\
& =\left|\int_{t_{2}}^{1} \frac{\gamma^{\prime \prime}(t)^{1 / 2+i \alpha}}{i\left(\xi+\eta \gamma^{\prime}(t)\right)} \frac{d}{d t}\left(e^{i(\xi t+\eta \gamma(t))}\right) d t\right| \\
& \leq \frac{\gamma^{\prime \prime}(1)^{1 / 2}}{\left|\xi+\eta \gamma^{\prime}(1)\right|}+\frac{\gamma^{\prime \prime}\left(t_{2}\right)^{1 / 2}}{\left|\xi+\eta \gamma^{\prime}\left(t_{2}\right)\right|}+\int_{t_{2}}^{1}\left|\left(\frac{\gamma^{\prime \prime}(t)^{1 / 2+i \alpha}}{\xi+\eta \gamma^{\prime}(t)}\right)^{\prime}\right| d t \\
& \leq \frac{C}{|\eta|}\left\{1+\frac{\gamma^{\prime \prime}\left(t_{2}\right)^{1 / 2}}{\gamma^{\prime}\left(t_{2}\right)}+(1+|\alpha|) \int_{t_{2}}^{1} \frac{\gamma^{\prime \prime \prime}(t)}{\gamma^{\prime \prime}(t)^{1 / 2} \gamma^{\prime}(t)} d t\right. \\
& \left.+\int_{t_{2}}^{1}|\eta|^{2} \frac{\gamma^{\prime \prime}(t)^{3 / 2}}{\left|\xi+\eta \gamma^{\prime}(t)\right|^{2}} d t\right\} \\
& \leq \frac{C}{|\eta| \gamma\left(t_{2}\right)^{1 / 2}}+C(1+|\alpha|) \frac{1}{|\eta|} \int_{t_{2}}^{1} \frac{\gamma^{\prime \prime}(t)^{3 / 2}}{\gamma^{\prime}(t)^{2}} d t \\
& \leq \frac{C}{|\eta|^{1 / 2}}+C(1+|\alpha|) \frac{1}{|\eta|} \int_{t_{2}}^{1} \frac{\gamma^{\prime}(t)}{\gamma(t)^{3 / 2}} d t \\
& \leq \frac{C}{|\eta|^{1 / 2}}+C(1+|\alpha|) \frac{1}{|\eta|} \int_{\gamma^{-1}\left(\frac{1}{|\eta|}\right)}^{1} \frac{\gamma^{\prime}(t)}{\gamma(t)^{3 / 2}} d t \\
& \leq \frac{C(1+|\alpha|)}{|\eta|^{1 / 2}}
\end{aligned}
$$


We now turn to the proof of the converse statement. By (9) under our assumptions on $\gamma$, the decay estimate gives

$$
\begin{gathered}
\int_{0}^{\delta} \frac{\gamma^{\prime \prime}(t)^{1 / 2}}{\gamma^{\prime}(t)} \gamma^{\prime}(t) d t \leq C \gamma(\delta)^{1 / 2} \\
\Longrightarrow \frac{\gamma^{\prime \prime}(\delta)^{1 / 2}}{\gamma^{\prime}(\delta)} \gamma(\delta) \leq C \gamma(\delta)^{1 / 2},
\end{gathered}
$$

which gives the stated inequality.

\section{Decay estimates for $n=3$}

Examples of failure of decay with mitigating factor for finite-type convex hypersurfaces in higher dimensions are given in $[6]$. In the radial setting Schulz, [18], showed that decay with mitigating factor $K_{\Gamma}^{1 / 2}$ fails for the example $\gamma(r)=e^{-1 / r}$ for odd dimensions. We begin, in Theorem 4.1, by extending this example in three dimensions. By considering the curves $\gamma(r)=e^{-1 / r^{m}}$ we show that decay can fail for mitigating factors $K_{\Gamma}^{\beta}$, for all $\frac{1}{3}<\beta \leq \frac{1}{2}$. Of course, for $n \geq 3$, failure of decay tells us nothing about restriction.

Before proceeding we note that if $\gamma$ is defined for $0<|t| \leq a$ and we change to polar coordinates then the decay estimates we are considering are of the form

$$
\left|\int_{0}^{a} e^{i \xi_{3} \gamma(r)} J_{0}\left(r\left|\xi^{\prime}\right|\right) K_{\Gamma}(r)^{\beta+i \alpha} r d r\right| \leq \frac{C(1+|\alpha|)^{N}}{\left|\xi_{3}\right|^{2 \beta}},
$$

where $\xi^{\prime}=\left(\xi_{1}, \xi_{2}\right)$ and $J_{0}$ is the Bessel function of order zero.

Theorem 4.1. If $\gamma(r)=e^{-\frac{1}{r^{m}}}$ and $\beta \geq \frac{m+2}{3 m+4}$ then, for $\left|\xi_{3}\right| \geq \frac{100}{\gamma(1 / 2)}$,

$$
\left|\int_{0}^{1 / 2} e^{i \xi_{3} \gamma(r)} K_{\Gamma}(r)^{\beta} r d r\right| \geq \frac{C}{\left|\xi_{3}\right|^{2 \beta}}\left(\log \left|\xi_{3}\right|\right)^{\frac{(3 m+4) \beta-(m+2)}{m}} .
$$

It follows that, for each $\frac{1}{3}<\beta \leq \frac{1}{2}$ there is a convex $\gamma \in C^{\infty}[0,1 / 2]$ with $\gamma(0)=\gamma^{\prime}(0)=0$ such that (11) fails.

We note that this is in contrast to the two-dimensional setting where decay with $\beta=\frac{1}{2}$ holds for the curve $\left(t, e^{-\frac{1}{t^{m}}}\right.$ ). (See Remark 2 after Proposition 3.1.) We also recall that in two dimensions we had no such results for $\beta<1 / 2$. (See the comments after the proof of Theorem 3.1.) Before proving Theorem 4.1 we need the following lemma. 
Lemma 4.1. Suppose $f \geq 0, f^{\prime} \leq 0, f^{\prime \prime} \geq 0$ on $[0, b]$ and $b \geq \frac{2 \pi}{\eta}$. Then

$$
\left|\int_{0}^{b} e^{i \eta t} f(t) d t\right| \geq \frac{C}{|\eta|}\left[f\left(\frac{2 \pi}{\eta}\right)-f\left(\frac{2 \pi\left[\frac{\eta b}{2 \pi}\right]}{\eta}\right)\right],
$$

where $[x]$ denotes the fractional part of $x$.

Proof: A change of variables shows that it is equivalent to show that, for $b>\frac{1}{\eta}$,

$$
\left|\int_{0}^{b} e^{2 \pi i \eta t} f(t) d t\right| \geq \frac{C}{|\eta|}\left[f\left(\frac{1}{\eta}\right)-f\left(\frac{[\eta b]}{\eta}\right)\right] .
$$

We start by noting that

$$
\begin{aligned}
\left|\int_{0}^{b} e^{2 \pi i \eta t} f(t) d t\right| \geq & \left|\int_{0}^{b} \sin (2 \pi \eta t) f(t) d t\right| \\
= & \frac{1}{\eta}\left|\int_{0}^{\eta b} \sin (2 \pi u) f\left(\frac{u}{\eta}\right) d u\right| \\
= & \frac{1}{\eta} \mid \sum_{j=0}^{\mid \eta \eta b]-1} \int_{j}^{j+1} \sin (2 \pi u) f\left(\frac{u}{\eta}\right) d u \\
& +\int_{[\eta b]}^{\eta b} \sin (2 \pi u) f\left(\frac{u}{\eta}\right) d u \mid .
\end{aligned}
$$

Then

$$
\begin{aligned}
\int_{j}^{j+1} \sin (2 \pi u) f\left(\frac{u}{\eta}\right) d u= & -\frac{1}{2 \pi}\left[f\left(\frac{j+1}{\eta}\right)-f\left(\frac{j}{\eta}\right)\right] \\
& +\frac{1}{2 \pi \eta} \int_{j}^{j+1} \cos (2 \pi u) f^{\prime}\left(\frac{u}{\eta}\right) d u \\
= & -\frac{1}{2 \pi \eta} \int_{j}^{j+1}(1-\cos (2 \pi u)) f^{\prime}\left(\frac{u}{\eta}\right) d u \\
\geq & 0 .
\end{aligned}
$$


Also,

$$
\begin{aligned}
\int_{[\eta b]}^{\eta b} \sin (2 \pi u) f\left(\frac{u}{\eta}\right) d u= & -\frac{1}{2 \pi \eta} \int_{[\eta b]}^{\eta b}(1-\cos (2 \pi u)) f^{\prime}\left(\frac{u}{\eta}\right) d u \\
& +\frac{1}{2 \pi} f(b)(1-\cos (2 \pi \eta b) \\
\geq & 0 .
\end{aligned}
$$

Then, using $f^{\prime}$ increasing and the Mean Value Theorem, we have

$$
\begin{aligned}
\left|\int_{0}^{b} e^{2 \pi i \eta t} f(t) d t\right| & \geq-\frac{1}{2 \pi \eta^{2}} \sum_{j=0}^{[\eta b]-1} f^{\prime}\left(\frac{j+1}{\eta}\right) \int_{j}^{j+1}(1-\cos (2 \pi u)) d u \\
& =-\frac{1}{2 \pi \eta^{2}} \sum_{j=0}^{[\eta b]-1} f^{\prime}\left(\frac{j+1}{\eta}\right) \\
& \geq \frac{1}{2 \pi \eta} \sum_{j=0}^{[\eta b]-2}\left[f\left(\frac{j+1}{\eta}\right)-f\left(\frac{j+2}{\eta}\right)\right]-\frac{1}{2 \pi \eta^{2}} f^{\prime}\left(\frac{[\eta] b}{\eta}\right) \\
& =\frac{1}{2 \pi \eta}\left[f\left(\frac{1}{\eta}\right)-f\left(\frac{[\eta] b}{\eta}\right)\right]-\frac{1}{2 \pi \eta^{2}} f^{\prime}\left(\frac{[\eta] b}{\eta}\right) \\
& \geq \frac{1}{2 \pi \eta}\left[f\left(\frac{1}{\eta}\right)-f\left(\frac{[\eta] b}{\eta}\right)\right] .
\end{aligned}
$$

Proof of Theorem 4.1: For $\gamma(r)=e^{-\frac{1}{r^{m}}}$, $K_{\Gamma}(r)=\frac{m(m+1)}{r^{3 m+4}}\left(\frac{m}{m+1}-r^{m}\right) e^{-\frac{2}{r^{m}}} \geq 0$ for $0 \leq r \leq 1 / 2, m \geq 1$. Then if we take $f(u)=\left(\log \frac{1}{u}\right)^{\frac{(3 m+4) \beta-(m+2)}{m}}\left(\frac{m}{m+1}-\frac{1}{\log \frac{1}{u}}\right)^{\beta} \frac{1}{u^{1-2 \beta}}$ we have

$$
\left|\int_{0}^{1 / 2} e^{i \xi_{3} \gamma(r)} K(r)^{\beta} r d r\right|=\left|\int_{0}^{\gamma(1 / 2)} e^{i \xi_{3} u} f(u) d u\right| .
$$

The given $f$ satisfies the conditions of Lemma 4.1 if $\beta \geq \frac{m+2}{3 m+4}$. It follows that

$$
\left|\int_{0}^{1 / 2} e^{i \xi_{3} \gamma(r)} K(r)^{\beta} r d r\right| \geq \frac{C}{\left|\xi_{3}\right|^{2 \beta}}\left(\log \left|\xi_{3}\right|\right)^{\frac{(3 m+4) \beta-(m+2)}{m}}
$$

for $\left|\xi_{3}\right| \geq \frac{100}{\gamma(1 / 2)}$. 
We also remark that if we take $\gamma$ as in the proof of Theorem 3.1, i.e. $\gamma^{\prime \prime}(t)=\frac{1}{(1-t)^{2} \log ^{2} \frac{1}{1-t}}$, then one can verify that $\gamma^{\prime}(t)^{\beta} \geq C \gamma^{\prime \prime}(t)^{\frac{1}{2}-\beta}$ for $\frac{1}{3}<\beta \leq \frac{1}{2}$ and all $t \in[0,1]$, from which it follows that $\left|\int_{0}^{1} K(r)^{\beta} r d r\right| \leq$ $C$ must fail. This is enough to show that decay fails for this $\gamma$ also.

We now give the three-dimensional (radial) analogue of Proposition 3.1. First we suppose that $\gamma:[0,1] \longrightarrow \mathbf{R}$ is convex and $C^{2}(0,1)$ and decay holds with a constant independent of $\gamma$, for $\gamma$ and all of its dilates $\gamma(\delta \cdot)$, for $\delta \leq 1$. The relevant decay estimate is now for $0 \leq \beta \leq \frac{1}{2}$,

$$
\left|\int_{0}^{1} e^{i \xi_{3} \gamma(r)} J_{0}\left(r\left|\xi^{\prime}\right|\right) K(r)^{\beta+i \alpha} r d r\right| \leq \frac{C(1+|\alpha|)^{N}}{\left|\xi_{3}\right|^{2 \beta}}
$$

for all $\xi_{3}$. Then the argument used for (8) together with dilation shows that

$$
\int_{0}^{\delta} K(r)^{\beta} r d r \leq C \gamma(\delta)^{2 \beta}
$$

for all $\delta \leq 1$, with $C$ independent of $\gamma$. We have stated this for $\beta$ rather than $\beta=\frac{1}{2}$ since in this case the inequality (13) may be violated for values other than $\frac{1}{2}$.

Proposition 4.1. a) Suppose that $\gamma:[0,1] \longrightarrow \mathbf{R}$ satisfies $\gamma$ and $\gamma^{\prime}$ convex, $\gamma \in C^{2}(0,1], \gamma \in C^{3}(0,1), \gamma(0)=\gamma^{\prime}(0)=0$ and $\left(\frac{r \gamma^{\prime \prime}(r)}{\gamma^{\prime}(r)}\right)^{\prime} \leq 0$ for $r \in(0,1)$. If also $\gamma^{\prime \prime}(r) \leq C \frac{\gamma^{\prime}(r)}{r}$ and $\gamma^{\prime \prime \prime}(r) \leq C \frac{\gamma^{\prime \prime}(r)}{r}$ for $r \in(0,1)$, then

$$
\left|\int_{0}^{1} e^{i \xi_{3} \gamma(r)} J_{0}\left(r\left|\xi^{\prime}\right|\right) K(r)^{1 / 2+i \alpha} r d r\right| \leq \frac{C(1+|\alpha|)^{N}}{\left|\xi_{3}\right|},
$$

for all $\xi_{3}$, with $C$ independent of $\gamma$.

Conversely, if $\gamma:[0,1] \longrightarrow \mathbf{R}$ is convex and $C^{3}(0,1]$, $\left(\frac{r \gamma^{\prime \prime}(r)}{\gamma^{\prime}(r)}\right)^{\prime} \leq 0$ for $r \in(0,1)$ and (14) holds for $\gamma$ and all of its dilates $\gamma(\delta \cdot)$, for $\delta \leq 1$, then $\gamma^{\prime \prime}(r) \leq C \frac{\gamma^{\prime}(r)}{r}$, for all $0 \leq r \leq 1$, with $C$ independent of $\gamma$. 
b) Suppose that $\gamma:[0,1] \longrightarrow \mathbf{R}$ satisfies $\gamma$ and $\gamma^{\prime}$ convex, $\gamma \in C^{2}(0,1]$, $\gamma \in C^{3}(0,1), \gamma(0)=\gamma^{\prime}(0)=0$ and $\left(\frac{r \gamma^{\prime \prime}(r)}{\gamma^{\prime}(r)}\right)^{\prime} \leq 0$ for $r \in(0,1)$. If also, $\gamma^{\prime \prime}(r) \leq C \frac{\gamma^{\prime}(r)}{r}\left|\log ^{\mu} \frac{1}{\gamma(r)}\right|$, for $r \in(0,1)$ and some $\mu \geq 0$, then

$\left|\int_{0}^{1} e^{i \xi_{3} \gamma(r)} J_{0}\left(r\left|\xi^{\prime}\right|\right) K(r)^{1 / 2+i \alpha} r d r\right| \leq \frac{C(1+|\alpha|)^{N}}{\left|\xi_{3}\right|}\left|\log ^{\frac{\mu}{2}+1}\right| \xi_{3}||$, for all $\xi_{3}$.

Remarks. 1. In parallel with the two-dimensional case, the converse statement in a) is a consequence of (13) and in fact the condition $\gamma^{\prime \prime}(r) \leq C \frac{\gamma^{\prime}(r)}{r}$ is equivalent to (13), under the additional assumption $\left(\frac{r \gamma^{\prime \prime}(r)}{\gamma^{\prime}(r)}\right)^{\prime} \leq 0$.

2. If, in b), one adds the hypothesis that $\gamma^{\prime \prime \prime}(r) \leq C \frac{\gamma^{\prime \prime}(r)}{r}\left|\log ^{\mu} \frac{1}{\gamma(r)}\right|$ for some $\mu \geq 0$ then one can obtain a similar estimate but with a power of $\frac{3 \mu}{2}$ on the logarithm. See Proposition 5.1 below. Therefore, with this extra condition the power of the logarithm may be taken to be $\min \left\{\frac{3 \mu}{2}, \frac{\mu}{2}+1\right\}$.

3. For the example $\gamma(r)=e^{-\frac{1}{r^{m}}}$, one has $\frac{r \gamma^{\prime \prime}(r)}{\gamma^{\prime}(r)} \approx \frac{1}{r^{m}}$ and so the conditions of b) hold but those of a) fail. This is in contrast to what happens in two dimensions; the conditions of Proposition 3.1 are satisfied by this curve.

We shall prove only the converse in a) at this point, leaving the sufficient conditions for decay to be dealt with simultaneously with the higher-dimensional result in the following section.

Proof of the converse in Proposition 4.1a): Suppose that the stated decay estimate holds. Then, by (13) we have $\int_{0}^{\delta}\left(\frac{r \gamma^{\prime \prime}(r)}{\gamma^{\prime}(r)}\right)^{1 / 2} \gamma^{\prime}(r) d r \leq$ $C \gamma(\delta)$. Using the assumption that $\left(\frac{r \gamma^{\prime \prime}(r)}{\gamma^{\prime}(r)}\right)^{\prime} \leq 0$ gives a lower bound of $\left(\frac{\delta \gamma^{\prime \prime}(\delta)}{\gamma^{\prime}(\delta)}\right)^{1 / 2} \gamma(\delta)$ for the integral and this gives the result.

\section{Decay estimates for $n \geq 4$}

We consider radial hypersurfaces $\Gamma(t)=(t, \gamma(|t|))$ in $\mathbf{R}^{n}$, where $n \geq 4$. We recall that $K_{\Gamma}(t)=\gamma^{\prime \prime}(|t|)\left(\frac{\gamma^{\prime}(|t|)}{|t|}\right)^{n-2}$. 
Following Lemma 2.1 we want inequalities such as

$$
\left|\int_{|t| \leq 1} e^{(i \xi \cdot \Gamma(t))} \phi(t) K_{\Gamma}(t)^{\beta+i \alpha} d t\right| \leq \frac{C(1+|\alpha|)^{N}}{\left|\xi_{n}\right|^{(n-1) \beta}}
$$

for some $N>0$ and $0 \leq \beta \leq 1 / 2$ with $\beta=1 / 2$ being the key value. Without the smooth cut-off $\phi$, one cannot hope for this decay to hold in dimensions higher than 3 , even for the nicest possible $\gamma$, that is $\gamma(r)=r^{2}$. This is because one cannot achieve decay better than $\frac{1}{\left|\xi_{n}\right|}$ near 1 . It follows that the restriction results one can obtain via decay estimates must necessarily be of the form (2).

If we change to polar coordinates then (15) becomes

$$
\begin{aligned}
\left|\int_{0}^{1} e^{i \xi_{n} \gamma(r)} \phi(r) \frac{1}{\left(r\left|\xi^{\prime}\right|\right)^{\frac{n-3}{2}}} J_{\frac{n-3}{2}}\left(r\left|\xi^{\prime}\right|\right) K(r)^{\beta+i \alpha} r^{n-2} d r\right| \\
\leq \frac{C(1+|\alpha|)^{N}}{\left|\xi_{n}\right|^{(n-1) \beta}}
\end{aligned}
$$

where $\xi^{\prime}=\left(\xi_{1}, \xi_{2}, \ldots, \xi_{n-1}\right)$ and $J_{\frac{n-3}{2}}$ is the Bessel function of order $\frac{n-3}{2}$. Throughout this section $\phi$ will be a smooth cut-off which vanishes at 1 . It need not vanish at 0 .

Sufficient conditions for decay are given in the following proposition. We note that although our primary interest is now $n \geq 4$, this result is valid for $n \geq 3$.

Proposition 5.1. Suppose that $\gamma:[0,1] \longrightarrow \mathbf{R}$ satisfies $\gamma(0)=\gamma^{\prime}(0)=$ $0, \gamma \in C^{k+2}(0,1)$ where $k=\left[\frac{n}{2}\right]$. Suppose that $\gamma$ and $\gamma^{\prime}$ are convex.

$$
\begin{gathered}
\text { If }\left|\frac{r \gamma^{(i+1)}(r)}{\gamma^{(i)}(r)}\right| \leq C\left|\log ^{\mu} \frac{1}{\gamma(r)}\right| \text { for some } \mu \geq 0, i=0,1, \ldots, k+1 \text {, then } \\
\left|\int e^{i \xi_{n} \gamma(r)} \phi(r) \frac{1}{\left(r\left|\xi^{\prime}\right|\right)^{\frac{n-3}{2}}} J_{\frac{n-3}{2}}\left(r\left|\xi^{\prime}\right|\right) K(r)^{1 / 2+i \alpha} r^{n-2} d r\right| \\
\leq \frac{C(1+|\alpha|)^{N}}{\left|\xi_{n}\right|^{(n-1) / 2}}\left|\log ^{\mu\left(k+\frac{1}{2}\right)}\right| \xi_{n}|| .
\end{gathered}
$$

Remarks. 1. Conditions could also be formulated to give decay for $\beta<1 / 2$.

2. For the full restriction result one needs to take $\mu=0$; in this case the conditions on $\gamma$ are closely related to the finite-type condition. The lemma below clarifies this. First we recall (from [5]) that $(t, \gamma(|t|))$ is finite-type $m, m>1$, at 0 if $\gamma \in C^{\infty}$ is such that $\gamma^{\prime}(0)=0$ and $m$ is the least integer such that $\gamma^{(m)}(0) \neq 0$. 
Lemma 5.1. Suppose that $\gamma:[0, \infty) \longrightarrow \mathbf{R}$.

a) If $\gamma \in C^{\infty}, \gamma \geq 0, \gamma^{\prime} \geq 0$ and $\frac{t \gamma^{\prime}(t)}{\gamma(t)} \leq C$ then $\gamma$ is finite-type at 0 .

b) If $\gamma$ is finite-type $m$ at 0 then $\left|\frac{t \gamma^{(r+1)}(t)}{\gamma^{(r)}(t)}\right| \approx C$ for $r=0,1, \ldots, m-1$. If $\gamma \in C^{k}$ with $k>m$ then one also has $\left|\frac{t \gamma^{(r+1)}(t)}{\gamma^{(r)}(t)}\right| \leq C$ for $r=$ $m, \ldots, k-1$.

Proof: a) $\frac{t \gamma^{\prime}(t)}{\gamma(t)} \leq C \Longrightarrow \log \frac{\gamma(1)}{\gamma(t)} \leq \log \frac{1}{t^{c}} \Longrightarrow \gamma(t) \geq \gamma(1) t^{c} \Longrightarrow \gamma$ is finite-type, since $\gamma \in C^{\infty}$.

b) If $\gamma(0)=\gamma^{\prime}(0)=\cdots=\gamma^{(m-1)}(0)=0$ and $\gamma^{(m)}(0)>0$ then $\gamma^{(r)}(t) \approx t^{m-r} \gamma^{(m)}(0)+O\left(t^{m-r+1}\right)$ for $r=0,1, \ldots, m$ and $\gamma^{(r)}(t) \approx$ $\gamma^{(r)}(0)+O(t)$ for $r=m+1, \ldots, k$. The results follow easily.

We now turn to the proof of Proposition 5.1. We first need some preliminary work.

Lemma 5.2. Suppose $\left|\frac{r \gamma^{(i+1)}(r)}{\gamma^{(i)}(r)}\right| \leq C\left|\log ^{\mu} \frac{1}{\gamma(r)}\right|$ for $i=1,2, \ldots, m+1$ and some $\mu \geq 0$.

a) Then

$$
\left|\left(\frac{K^{\prime}}{K}\right)^{(m)}(r)\right| \leq \frac{C}{r^{m+1}}\left|\log ^{\mu(m+1)} \frac{1}{\gamma(r)}\right| .
$$

b) If also ||$\xi^{\prime}\left|+\xi_{n} \gamma^{\prime}(r)\right| \geq c\left|\xi_{n}\right| \gamma^{\prime}(r)$ then

$$
\left|\left(\frac{\xi_{n} \gamma^{\prime \prime}}{\left|\xi^{\prime}\right|+\xi_{n} \gamma^{\prime}}\right)^{(m)}(r)\right| \leq \frac{C}{r^{m+1}}\left|\log ^{\mu(m+1)} \frac{1}{\gamma(r)}\right| .
$$

Proof: A straightforward calculation.

We now define $D_{r}^{1} f(r)=\frac{d}{d r}\left(\frac{f(r)}{\left|\xi^{\prime}\right|+\xi_{n} \gamma^{\prime}(r)}\right) ; D_{r}^{k} f(r)=\frac{d}{d r}\left(\frac{D_{r}^{k-1} f(r)}{\left|\xi^{\prime}\right|+\xi_{n} \gamma^{\prime}(r)}\right)$ for $k \geq 2$.

Lemma 5.3. If $\left|\frac{r \gamma^{(i+1)}(r)}{\gamma^{(i)}(r)}\right| \leq C\left|\log ^{\mu} \frac{1}{\gamma(r)}\right|$ for $i=1,2, \ldots, m+1$ and some $\mu \geq 0$ and if ||$\xi^{\prime}\left|+\xi_{n} \gamma^{\prime}(r)\right| \geq c\left|\xi_{n}\right| \gamma^{\prime}(r)$, then for some $N>0$ and all $0 \leq \beta \leq 1 / 2$, we have, for $\nu \geq 0$ and $0<a \leq 1$,

$$
\begin{aligned}
& \left|D_{r}^{m}\left(K^{\beta+i \alpha}(r) r^{\nu} \phi\left(\frac{r}{a}\right)\right)\right| \\
\leq & \frac{C(1+|\alpha|)^{N}}{\left|\xi_{n}\right|^{m}} \gamma^{\prime}(r)^{(n-1) \beta-m} r^{\nu-(n-1) \beta}\left|\frac{1}{r} \log ^{\mu} \frac{1}{\gamma(r)}+\frac{1}{a}\right|^{m}\left|\log ^{\mu / 2} \frac{1}{\gamma(r)}\right| .
\end{aligned}
$$


Proof: Let $H_{k}(r)=(\beta+i \alpha) \frac{K^{\prime}(r)}{K(r)}+\frac{\nu}{r}-k \frac{\xi_{n} \gamma^{\prime \prime}(r)}{\left|\xi^{\prime}\right|+\xi_{n} \gamma^{\prime}(r)}$ and then observe that

$$
D_{r}^{1}\left(K^{\beta+i \alpha}(r) r^{\nu} \phi\left(\frac{r}{a}\right)\right)=\frac{K(r)^{\beta+i \alpha} r^{\nu}}{\left|\xi^{\prime}\right|+\xi_{n} \gamma^{\prime}(r)}\left(\frac{1}{a} \phi^{\prime}\left(\frac{r}{a}\right)+\phi\left(\frac{r}{a}\right) H_{1}(r)\right)
$$

and

$$
\begin{aligned}
D_{r}^{2}\left(K^{\beta+i \alpha}(r) r^{\nu} \phi\left(\frac{r}{a}\right)\right) & \\
=\frac{K(r)^{\beta+i \alpha} r^{\nu}}{\left(\left|\xi^{\prime}\right|+\xi_{n} \gamma^{\prime}(r)\right)^{2}} & \left(\left(\frac{1}{a} \phi^{\prime}\left(\frac{r}{a}\right)+\phi\left(\frac{r}{a}\right) H_{1}(r)\right)^{\prime}\right. \\
& \left.+\left(\frac{1}{a} \phi^{\prime}\left(\frac{r}{a}\right)+\phi\left(\frac{r}{a}\right) H_{1}(r)\right) H_{2}(r)\right) .
\end{aligned}
$$

In general one finds

$$
D_{r}^{m}\left(K^{\beta+i \alpha}(r) r^{\nu} \phi\left(\frac{r}{a}\right)\right)=\frac{K(r)^{\beta+i \alpha} r^{\nu}}{\left(\left|\xi^{\prime}\right|+\xi_{n} \gamma^{\prime}(r)\right)^{m}} F_{m}(r)
$$

where $F_{m}(r)$ is an algebraic expression in $\phi, \phi^{\prime}, \ldots, \phi^{(m)}, H_{0}, H_{0}^{\prime}, \ldots, H_{0}^{(m-1)}, H_{1}, H_{1}^{\prime}, \ldots, H_{1}^{(m-2)}, \ldots, H_{m-1}$.

Using Lemma 5.2 we see that, for some $N>0$, $\left|F_{m}(r)\right| \leq C(1+|\alpha|)^{N}\left|\frac{1}{r} \log ^{\mu} \frac{1}{\gamma(r)}+\frac{1}{a}\right|^{m}$ and so using again the assumption that ||$\xi^{\prime}\left|+\xi_{n} \gamma^{\prime}(r)\right| \geq c\left|\xi_{n}\right| \gamma^{\prime}(r)$ we have

$$
\begin{aligned}
& \left|D_{r}^{m}\left(K^{\beta+i \alpha}(r) r^{\nu} \phi\left(\frac{r}{a}\right)\right)\right| \\
& \leq C(1+|\alpha|)^{N} \frac{K(r)^{\beta} r^{\nu}}{\left|\xi_{n} \gamma^{\prime}(r)\right|^{m}}(r)\left|\frac{1}{r} \log ^{\mu} \frac{1}{\gamma(r)}+\frac{1}{a}\right|^{m} \\
& =\frac{C(1+|\alpha|)^{N}}{\left|\xi_{n}\right|^{m}} \gamma^{\prime \prime}(r)^{\beta} \gamma^{\prime}(r)^{(n-2) \beta-m} r^{\nu-(n-2) \beta}\left|\frac{1}{r} \log ^{\mu} \frac{1}{\gamma(r)}+\frac{1}{a}\right|^{m} \\
& \leq \frac{C(1+|\alpha|)^{N}}{\left|\xi_{n}\right|^{m}} \gamma^{\prime}(r)^{(n-1) \beta-m} r^{\nu-(n-1) \beta}\left|\frac{1}{r} \log ^{\mu} \frac{1}{\gamma(r)}+\frac{1}{a}\right|^{m}\left|\log ^{\mu / 2} \frac{1}{\gamma(r)}\right| .
\end{aligned}
$$


Proof of Propositions 5.1 and 4.1b): In both propositions the integral over $\left[0, \gamma^{-1}\left(1 /\left|\xi_{n}\right|\right)\right]$ is dealt with via a size estimate. We first note that if $a<e^{-1}$ and $\alpha>0$ then

$$
\int_{0}^{a} \log ^{\alpha} \frac{1}{u} d u \leq C_{\alpha} a \log ^{\alpha} \frac{1}{a} .
$$

To see this we integrate by parts:

$$
\int_{0}^{a} \log ^{\alpha} \frac{1}{u} d u=a \log ^{\alpha} \frac{1}{a}+\alpha \int_{0}^{a} \log ^{\alpha-1} \frac{1}{u} d u .
$$

If $\alpha \leq 1$ then $\log ^{\alpha-1} \frac{1}{u} \leq \log ^{\alpha-1} \frac{1}{a}$ for all $u \in(0, a)$ and since $a<e^{-1}$ this is bounded by $\log ^{\alpha} \frac{1}{a}$. This then gives

$$
\int_{0}^{a} \log ^{\alpha} \frac{1}{u} d u \leq(1+\alpha) a \log ^{\alpha} \frac{1}{a} .
$$

If $\alpha>1$, we integrate by parts repeatedly until the logarithm in the integrand has a negative exponent and then argue in a similar way.

We now consider

$$
\begin{aligned}
\int_{0}^{\gamma^{-1}\left(1 /\left|\xi_{n}\right|\right)} & K(r)^{1 / 2} r^{n-2} d r \\
& =\int_{0}^{\gamma^{-1}\left(1 /\left|\xi_{n}\right|\right)} \gamma^{\prime \prime}(r)^{1 / 2}\left(\frac{\gamma^{\prime}(r)}{r}\right)^{(n-2) / 2} r^{n-2} d r \\
& \leq C \int_{0}^{\gamma^{-1}\left(1 /\left|\xi_{n}\right|\right)} \gamma^{\prime}(r)^{(n-1) / 2}\left|\log ^{\mu / 2} \frac{1}{\gamma(r)}\right| r^{(n-3) / 2} d r
\end{aligned}
$$

using the assumption that $\gamma^{\prime \prime}(r) \leq C \frac{\gamma^{\prime}(r)}{r}\left|\log ^{\mu} \frac{1}{\gamma(r)}\right|$.

If $n=3$, this is

$$
\int_{0}^{\gamma^{-1}\left(1 /\left|\xi_{3}\right|\right)} \gamma^{\prime}(r)\left|\log ^{\mu / 2} \frac{1}{\gamma(r)}\right| d r=\int_{0}^{\frac{1}{\left|\xi_{3}\right|}}\left|\log ^{\mu / 2} \frac{1}{u}\right| d u .
$$

For $\frac{1}{\left|\xi_{3}\right|}<e^{-1}$, we can apply (17) to obtain a bound of $\frac{C_{\mu}}{\left|\xi_{3}\right|}\left|\log ^{\mu / 2}\right| \xi_{3}||$. In all dimensions, for $\frac{1}{\left|\xi_{n}\right|} \geq e^{-1}$, we have the trivial estimate

$$
\int_{0}^{\gamma^{-1}\left(\frac{1}{\left|\xi_{n}\right|}\right)} K(r)^{1 / 2} r^{n-2} d r \leq C \leq \frac{C}{\left|\xi_{n}\right|}\left|\log ^{\mu / 2}\right| \xi_{n}|| .
$$


We now consider the case $n \geq 4$. From the argument above we see that we may assume that $\frac{1}{\left|\xi_{n}\right|}<e^{-1}$. Then we use the additional assumption that $\gamma^{\prime}(r) \leq C \frac{\gamma(r)}{r}\left|\log ^{\mu} \frac{1}{\gamma(r)}\right|$, to obtain

$$
\begin{aligned}
& \int_{0}^{\gamma^{-1}\left(1 /\left|\xi_{n}\right|\right)}\left(\frac{\gamma^{\prime}(r)}{r}\right)^{\frac{n-1}{2}}\left|\log ^{\mu / 2}\left(\frac{1}{\gamma(r)}\right)\right| r^{n-2} d r \\
& \quad \leq \int_{0}^{\gamma^{-1}\left(1 /\left|\xi_{n}\right|\right)}\left(\frac{\gamma(r)}{r}\right)^{(n-3) / 2} \gamma^{\prime}(r)\left|\log ^{\mu\left(\frac{n-2}{2}\right)}\left(\frac{1}{\gamma(r)}\right)\right| r^{(n-3) / 2} d r \\
& \quad=\int_{0}^{\frac{1}{\xi_{n} \mid}} u^{\frac{n-3}{2}}\left|\log ^{\mu\left(\frac{n-2}{2}\right)} \frac{1}{u}\right| d u \\
& \quad \leq \frac{C_{\mu}}{\left|\xi_{n}\right|^{(n-3) / 2}} \cdot \frac{1}{\left|\xi_{n}\right|}\left|\log ^{\mu\left(\frac{n-2}{2}\right)}\right| \xi_{n}||,
\end{aligned}
$$

by (17), which gives us the desired estimate.

For the integral over $\left[\gamma^{-1}\left(1 /\left|\xi_{n}\right|\right), 1\right]$ there are several cases to consider. We shall assume that $\left|\xi^{\prime}\right| \geq 1$ since the argument simplifies otherwise. This gives us two cases to consider.

Case 1: $\gamma^{-1}\left(\frac{1}{\left|\xi_{n}\right|}\right)<\frac{1}{\left|\xi^{\prime}\right|}$.

In this case we take $\phi_{1}$ to be a smooth cut-off which takes the value 1 on $\left[0, \frac{1}{2}\right]$ and vanishes at 1 . Then we need to estimate

$$
\left|\int_{\gamma^{-1}\left(\frac{1}{\left|\xi_{n}\right|}\right)}^{\frac{2}{\left|\xi^{\prime}\right|}} e^{i \xi_{n} \gamma(r)} \phi(r) \phi_{1}\left(\frac{r\left|\xi^{\prime}\right|}{2}\right) \frac{1}{\left(r\left|\xi^{\prime}\right|\right)^{\frac{n-3}{2}}} J_{\frac{n-3}{2}}\left(r\left|\xi^{\prime}\right|\right) K(r)^{\frac{1}{2}+i \alpha} r^{n-2} d r\right|
$$

and

$$
\left|\int_{\frac{1}{\left|\xi^{\prime}\right|}}^{1} e^{i \xi_{n} \gamma(r)} \phi(r)\left(1-\phi_{1}\left(\frac{r\left|\xi^{\prime}\right|}{2}\right)\right) \frac{1}{\left(r\left|\xi^{\prime}\right|\right)^{\frac{n-3}{2}}} J_{\frac{n-3}{2}}\left(r\left|\xi^{\prime}\right|\right) K(r)^{\frac{1}{2}+i \alpha} r^{n-2} d r\right| .
$$

Here and throughout this proof we shall replace the Bessel function $J_{\frac{n-3}{2}}$ by the leading term in its expansion. The remainders are dealt with by similar arguments. See [26, Section 7.21 and Section 3.1] 
for the asymptotic expansion and the series expansion for small argument respectively. Thus, instead of bounding (18) and (19), we shall bound instead

$$
\left|\int_{\gamma^{-1}\left(\frac{1}{\left|\xi_{n}\right|}\right)}^{\frac{2}{\left|\xi^{\prime}\right|}} e^{i \xi_{n} \gamma(r)} \phi_{1}\left(\frac{r\left|\xi^{\prime}\right|}{2}\right) K(r)^{\frac{1}{2}+i \alpha} r^{n-2} d r\right|
$$

and

$$
\frac{1}{\left|\xi^{\prime}\right|^{\frac{n-2}{2}}}\left|\int_{\frac{1}{\left|\xi^{\prime}\right|}}^{1} e^{i\left(\xi_{n} \gamma(r)+r\left|\xi^{\prime}\right|\right)} \phi(r)\left(1-\phi_{1}\left(\frac{r\left|\xi^{\prime}\right|}{2}\right)\right) K(r)^{\frac{1}{2}+i \alpha} r^{\frac{n-2}{2}} d r\right|
$$

We consider (20) for Proposition 4.1b) $(n=3)$ first. We remind the reader that the conditions are slightly different from those in Proposition 5.1. In particular $\left(\frac{r \gamma^{\prime \prime}}{\gamma^{\prime}}\right)$ is assumed to be decreasing. For notational simplicity we let $a=\gamma^{-1}\left(\frac{1}{\left|\xi_{3}\right|}\right)$. Integrating by parts once gives a bound of

$$
\frac{C}{\left|\xi_{3}\right|}\left\{\frac{K^{1 / 2}(a) a}{\gamma^{\prime}(a)}+\int_{a}^{\frac{2}{\left|\xi^{\prime}\right|}}\left|\frac{d}{d r}\left[\frac{\phi_{1}\left(\frac{r\left|\xi^{\prime}\right|}{2}\right) K(r)^{1 / 2+i \alpha} r}{\gamma^{\prime}(r)}\right]\right| d r\right\} .
$$

Our assumption that $\frac{r \gamma^{\prime \prime}(r)}{\gamma^{\prime}(r)} \leq C\left|\log ^{\mu} \frac{1}{\gamma(r)}\right| \operatorname{gives} \frac{K^{1 / 2}(a) a}{\gamma^{\prime}(a)} \leq C\left|\log ^{\mu / 2}\right| \xi_{3}||$. For the integral we also use the monotonicity condition on $\frac{r \gamma^{\prime \prime}}{\gamma^{\prime}}$ to obtain a bound of

$$
\begin{aligned}
& \frac{C(1+|\alpha|)}{\left|\xi_{3}\right|}\left\{\int_{a}^{\frac{2}{\left|\xi^{\prime}\right|}}\left|\xi^{\prime}\right|\left|\phi_{1}^{\prime}\left(\frac{r\left|\xi^{\prime}\right|}{2}\right)\right| \frac{K(r)^{1 / 2} r}{\gamma^{\prime}(r)} d r\right. \\
& \left.+\int_{a}^{\frac{2}{\left|\xi^{\prime}\right|}} \phi_{1}\left(\frac{r\left|\xi^{\prime}\right|}{2}\right)\left[\frac{K^{\prime}(r)}{K(r)}+\frac{\gamma^{\prime \prime}(r)}{\gamma^{\prime}(r)}+\frac{1}{r}\right] \frac{K(r)^{1 / 2} r}{\gamma^{\prime}(r)} d r\right\} \\
& \leq \frac{C(1+|\alpha|)}{\left|\xi_{3}\right|}\left\{\frac{K(a)^{1 / 2} a}{\gamma^{\prime}(a)}\left(1+\int_{a}^{\frac{2}{\left|\xi^{\prime}\right|}} \frac{\gamma^{\prime \prime}(r)}{\gamma^{\prime}(r)} d r\right)\right. \\
& \left.+\int_{a}^{\frac{2}{\left|\xi^{\prime}\right|}} \frac{K^{\prime}(r)}{K(r)^{1 / 2}} \frac{r}{\gamma^{\prime}(r)} d r\right\} .
\end{aligned}
$$


We now note that integrating by parts we obtain

$$
\begin{aligned}
\int_{a}^{\frac{2}{\left|\xi^{\prime}\right|}} \frac{K^{\prime}(r)}{K(r)^{1 / 2}} \frac{r}{\gamma^{\prime}(r)} d r & \leq \int_{a}^{\frac{2}{\left|\xi^{\prime}\right|}}\left(\frac{\gamma^{\prime \prime}(r)}{\gamma^{\prime}(r)}+\frac{1}{r}\right) \frac{K(r)^{1 / 2} r}{\gamma^{\prime}(r)} d r \\
& \leq C \frac{K(a)^{1 / 2} a}{\gamma^{\prime}(a)} \int_{a}^{\frac{2}{\left|\xi^{\prime}\right|}} \frac{\gamma^{\prime \prime}(r)}{\gamma^{\prime}(r)} d r
\end{aligned}
$$

So we finally obtain a bound of $\frac{C(1+|\alpha|)}{\left|\xi_{3}\right|}\left|\log ^{\mu / 2}\right| \xi_{3}||\left\{1+\left|\log \frac{\gamma^{\prime}\left(\frac{2}{\left|\xi^{\prime}\right|}\right)}{\gamma^{\prime}(a)}\right|\right\}$, which gives the estimate $\frac{C(1+|\alpha|)}{\left|\xi_{3}\right|}\left|\log ^{\frac{\mu}{2}+1}\right| \xi_{3}||$, since we have the trivial estimate $\frac{\gamma^{\prime}\left(\frac{2}{\mid \xi^{\prime}}\right)}{\gamma^{\prime}(a)} \leq \frac{C}{\gamma^{\prime}(a)} \leq C\left|\xi_{3}\right|$.

We now estimate (20) under the conditions for Proposition 5.1. First we define $\tilde{D}_{r}^{k}$ exactly as we $\operatorname{did} D_{r}^{k}$ but with $\left|\xi^{\prime}\right|=0$. We again take $a=\gamma^{-1}\left(\frac{1}{\left|\xi_{n}\right|}\right)$. Integrating by parts repeatedly leads to an upper bound of

$$
\begin{aligned}
\frac{K(a)^{1 / 2} a^{n-2}}{\left|\xi_{n}\right| \gamma^{\prime}(a)}+ & \sum_{i=1}^{k-1} \frac{1}{\left|\xi_{n}\right| \gamma^{\prime}(a)}\left|\tilde{D}_{r}^{i}\left[\phi_{1}\left(\frac{r\left|\xi^{\prime}\right|}{2}\right) K(r)^{1 / 2+i \alpha} r^{n-2}\right](a)\right| \\
& +\left|\int_{a}^{\frac{2}{\left|\xi^{\prime}\right|}} e^{i \xi_{n} \gamma(r)} \tilde{D}_{r}^{k}\left[\phi_{1}\left(\frac{r\left|\xi^{\prime}\right|}{2}\right) K(r)^{1 / 2+i \alpha} r^{n-2}\right] d r\right| \\
=\mathcal{A}+\mathcal{B} & +\mathcal{C} .
\end{aligned}
$$

Using the assumptions that $\gamma^{\prime \prime}(r) \leq C \frac{\gamma^{\prime}(r)}{r}\left|\log ^{\mu} \frac{1}{\gamma(r)}\right|$ and $\gamma^{\prime}(r) \leq C \frac{\gamma(r)}{r}\left|\log ^{\mu} \frac{1}{\gamma(r)}\right|$ allows us to bound $\mathcal{A}$ as follows;

$$
\begin{aligned}
\mathcal{A} & \leq \frac{C}{\left|\xi_{n}\right|} \gamma^{\prime}(a)^{\frac{n-3}{2}} a^{\frac{n-3}{2}}\left|\log ^{\mu / 2}\right| \xi_{n}|| \\
& \leq \frac{C}{\left|\xi_{n}\right|} \gamma(a)^{\frac{n-3}{2}}\left|\log ^{\mu \frac{n-2}{2}}\right| \xi_{n}|| \\
& \leq \frac{C}{\left|\xi_{n}\right|^{\frac{n-1}{2}}}\left|\log ^{\mu \frac{n-2}{2}}\right| \xi_{n}|| .
\end{aligned}
$$


For $\mathcal{B}$ we use Lemma 5.3;

$$
\begin{aligned}
\mathcal{B} & \leq C(1+|\alpha|) \sum_{i=1}^{k-1} \frac{1}{\left|\xi_{n}\right|^{i+1}} \gamma^{\prime}(a)^{\frac{n-3}{2}-i} a^{\frac{n-3}{2}}\left|\frac{1}{a} \log ^{\mu}\right| \xi_{n}|+| \xi^{\prime}||^{i} \log ^{\mu / 2}\left|\xi_{n}\right| \\
& \leq C(1+|\alpha|) \frac{\left|\log ^{\mu / 2}\right| \xi_{n}||}{\left|\xi_{n}\right|^{\frac{n-1}{2}}} \sum_{i=1}^{k-1}\left|\log ^{\mu\left(\frac{n-3}{2}-i\right)}\right| \xi_{n}||\left|\log ^{\mu}\right| \xi_{n}|+| \xi^{\prime}|a|^{i} \\
& \leq C(1+|\alpha|) \frac{\left|\log ^{\mu\left(\frac{n-3}{2}\right)}\right| \xi_{n}||}{\left|\xi_{n}\right|^{\frac{n-1}{2}}} .
\end{aligned}
$$

Finally Van der Corput's lemma and Lemma 5.3 give

$$
\begin{aligned}
\mathcal{C} & \leq \frac{C(1+|\alpha|)^{N}\left|\log ^{\mu / 2}\right| \xi_{n}||}{\left|\xi_{n}\right|^{k+1} \gamma^{\prime}(a)} \max _{\left[a, \frac{2}{\left|\xi^{\prime}\right|}\right]} \gamma^{\prime}(r)^{\frac{n-1}{2}-k} r^{\frac{n-3}{2}}\left|\frac{1}{r} \log ^{\mu} \frac{1}{\gamma(r)}+\right| \xi^{\prime}||^{k} \\
& \leq \frac{C(1+|\alpha|)^{N}\left|\log ^{\mu(k+1 / 2)}\right| \xi_{n}||}{\left|\xi_{n}\right|^{k+1} \gamma^{\prime}(a)} \max _{\left[a, \frac{2}{\left|\xi^{\prime}\right|}\right]} \gamma^{\prime}(r)^{\frac{n-1}{2}-k} r^{\frac{n-3}{2}-k} .
\end{aligned}
$$

The required estimate follows on choosing $k=\left[\frac{n}{2}\right]$ and noting that $\gamma^{\prime}(a) \geq \gamma(a) / a$.

This completes the estimates for (20); we now turn to (21). How we proceed depends on whether or not the phase function has a critical point in the interval of integration.

We take first the case when $\left|\xi_{n} \gamma^{\prime}(r)+\right| \xi^{\prime}|| \geq c\left|\xi_{n} \gamma^{\prime}(r)\right|$ for all $r \in$ $\left[\frac{1}{\left|\xi^{\prime}\right|}, 1\right]$. As before we shall consider the three-dimensional case first. In this case $\phi(r) \equiv 1$ on the interval of integration. We note that the cut-off $\left[1-\phi_{1}\left(\frac{r\left|\xi^{\prime}\right|}{2}\right)\right]$ vanishes at $\frac{1}{\left|\xi^{\prime}\right|}$.

Integrating (21) by parts once gives a bound of

$$
\frac{C}{\left|\xi_{3}\right|\left|\xi^{\prime}\right|^{1 / 2}} \frac{K^{1 / 2}(1)}{\gamma^{\prime}(1)}+\frac{C}{\left|\xi^{\prime}\right|^{1 / 2}} \int_{\frac{1}{\left|\xi^{\prime}\right|}}^{1}\left|\frac{d}{d r}\left[\frac{\left(1-\phi_{1}\left(\frac{r\left|\xi^{\prime}\right|}{2}\right)\right) K(r)^{1 / 2+i \alpha} r^{1 / 2}}{\xi_{3} \gamma^{\prime}(r)+\left|\xi^{\prime}\right|}\right]\right| d r
$$


The estimate of the integral is similar to that needed for the integral over $\left[a, \frac{2}{\left|\xi^{\prime}\right|}\right]$. With our assumptions on $\gamma$ we have

$$
\begin{gathered}
\frac{C(1+|\alpha|))}{\left|\xi_{3}\right|\left|\xi^{\prime}\right|^{1 / 2}}\left\{\int_{\frac{1}{\left|\xi^{\prime}\right|}}^{1} \frac{\left|\xi^{\prime}\right|}{r^{1 / 2}}\left|\phi_{1}^{\prime}\left(\frac{r\left|\xi^{\prime}\right|}{2}\right)\right| \frac{K(r)^{1 / 2} r}{\gamma^{\prime}(r)} d r\right. \\
\left.\quad+\int_{\frac{1}{\left|\xi^{\prime}\right|}}^{1} \frac{\left(1-\phi_{1}\left(\frac{r\left|\xi^{\prime}\right|}{2}\right)\right)}{r^{1 / 2}}\left[\frac{K^{\prime}(r)}{K(r)}+\frac{\gamma^{\prime \prime}(r)}{\gamma^{\prime}(r)}+\frac{1}{r}\right] \frac{K(r)^{1 / 2} r}{\gamma^{\prime}(r)} d r\right\} \\
\leq \frac{C(1+|\alpha|)}{\left|\xi_{3}\right|} \frac{K\left(\frac{1}{\left|\xi^{\prime}\right|}\right)^{1 / 2} \frac{1}{\left|\xi^{\prime}\right|}}{\gamma^{\prime}\left(\frac{1}{\left|\xi^{\prime}\right|}\right)}\left\{1+\int_{\frac{1}{\left|\xi^{\prime}\right|}}^{1} \frac{\gamma^{\prime \prime}(r)}{\gamma^{\prime}(r)} d r\right\} \\
\leq \frac{C(1+|\alpha|)}{\left|\xi_{3}\right|}\left|\log ^{\mu / 2}\right| \xi_{3}||\left\{1+\log \frac{\gamma^{\prime}(1)}{\gamma^{\prime}\left(\frac{1}{\left|\xi^{\prime}\right|}\right)}\right\},
\end{gathered}
$$

which gives the required estimate.

For $n \geq 4$ we integrate by parts repeatedly again, noting that this time the cut-off vanishes both at 1 and $\frac{1}{\left|\xi^{\prime}\right|}$. This replaces (21) by

$$
\frac{1}{\left|\xi^{\prime}\right|^{\frac{n-2}{2}}}\left|\int_{\frac{1}{\left|\xi^{\prime}\right|}}^{1} e^{i\left(\xi_{n} \gamma(r)+r\left|\xi^{\prime}\right|\right)} D_{r}^{k}\left[\phi(r)\left(1-\phi\left(\frac{r\left|\xi^{\prime}\right|}{2}\right)\right) K(r)^{1 / 2+i \alpha} r^{\frac{n-2}{2}}\right] d r\right| \text {. }
$$

Van der Corput's lemma and Lemma 5.3 allow us to bound this by

$$
\begin{gathered}
\frac{C(1+|\alpha|)^{N}\left|\log ^{\mu(k+1 / 2)}\right| \xi_{n}||}{\left|\xi^{\prime}\right|^{\frac{n-2}{2}}\left|\xi^{\prime}\right|\left|\xi_{n}\right|^{k}} \max _{\left[\frac{1}{\left|\xi^{\prime}\right|}, 1\right]} \frac{\gamma^{\prime}(r)^{\frac{n-1}{2}-k}}{r^{1 / 2+k}} \\
\leq \frac{C(1+|\alpha|)^{N}\left|\log ^{\mu(k+1 / 2)}\right| \xi_{n}||}{\left|\xi_{n}\right|^{k}\left|\xi^{\prime}\right|^{\frac{n-1}{2}-k}} \max _{\left[\frac{1}{\left|\xi^{\prime}\right|}, 1\right]} \gamma^{\prime}(r)^{\frac{n-1}{2}-k} .
\end{gathered}
$$

The required estimate follows on choosing $k=\left[\frac{n}{2}\right]$.

Next we suppose that the phase function has a critical point in $\left[\frac{1}{\left|\xi^{\prime}\right|}, 1\right]$. So we take $t_{0}$ such that $\gamma^{\prime}\left(t_{0}\right)=\left|\frac{\xi^{\prime}}{\xi_{n}}\right|$. We also take $t_{1}$ and $t_{2}$ to be such that $\gamma^{\prime}\left(t_{1}\right)=\frac{1}{2}\left|\frac{\xi^{\prime}}{\xi_{n}}\right|$ and $\gamma^{\prime}\left(t_{2}\right)=2\left|\frac{\xi^{\prime}}{\xi_{n}}\right|$ and we assume that $\frac{1}{\left|\xi^{\prime}\right|}<t_{1}<$ $t_{0}<t_{2} \leq 1$. Of course, it could be that $t_{1}$ and/or $t_{2}$ lie outside the interval of integration, however these cases can be treated in a similar manner. 
For all dimensions $n \geq 3$ we use Van der Corput's lemma to give

$$
\begin{aligned}
\frac{1}{\left|\xi^{\prime}\right|^{\frac{n-2}{2}}} \mid \int_{\frac{1}{\left|\xi^{\prime}\right|}}^{t_{1}} & e^{i\left(\xi_{n} \gamma(r)+\left|\xi^{\prime}\right| r\right)} \phi(r)\left(1-\phi\left(\frac{r\left|\xi^{\prime}\right|}{2}\right)\right) K(r)^{1 / 2+i \alpha} r^{\frac{n-2}{2}} d r \mid \\
& \leq \frac{C(1+|\alpha|)}{\left|\xi^{\prime}\right|^{\frac{n-2}{2}}} \frac{1}{\left|\xi^{\prime}\right|} K\left(t_{1}\right)^{1 / 2} t_{1}^{\frac{n-2}{2}} \\
& \leq \frac{C(1+|\alpha|)}{\left|\xi^{\prime}\right|^{\frac{n}{2}}} \gamma^{\prime \prime}\left(t_{1}\right)^{1 / 2} \gamma^{\prime}\left(t_{1}\right)^{\frac{n-2}{2}} \\
& =\frac{C(1+|\alpha|)}{\left|\xi^{\prime}\right|^{\frac{n}{2}}}\left(\frac{t_{1} \gamma^{\prime \prime}\left(t_{1}\right)}{\gamma^{\prime}\left(t_{1}\right)}\right)^{1 / 2} \frac{\gamma^{\prime}\left(t_{1}\right)^{\frac{n-1}{2}}}{t_{1}^{1 / 2}} \\
& \leq \frac{C(1+|\alpha|)}{\left|\xi^{\prime}\right|^{\frac{n}{2}}}\left|\frac{\xi^{\prime}}{\xi_{n}}\right|^{\frac{n-1}{2}}\left|\xi^{\prime}\right|^{1 / 2}\left|\log ^{\mu / 2}\right| \xi_{n}|| \\
& \leq \frac{C(1+|\alpha|)}{\left|\xi_{n}\right|^{\frac{n-1}{2}}}\left|\log ^{\mu / 2}\right| \xi_{n}|| .
\end{aligned}
$$

Again, for all dimensions $n \geq 3$, for the integral over $\left[t_{1}, t_{2}\right]$ we use convexity of $\gamma$ and $\gamma^{\prime}$ together with Van der Corput with the second derivative to obtain

$$
\begin{aligned}
& \frac{1}{\left|\xi^{\prime}\right|^{\frac{n-2}{2}} \mid \int_{t_{1}}^{t_{2}}} e^{i\left(\xi_{n} \gamma(r)+\left|\xi^{\prime}\right| r\right)} \phi(r)\left(1-\phi\left(\frac{r\left|\xi^{\prime}\right|}{2}\right)\right) K(r)^{1 / 2+i \alpha} r^{\frac{n-2}{2}} d r \mid \\
& \quad \leq \frac{C(1+|\alpha|)}{\left|\xi^{\prime}\right|^{\frac{n-2}{2}}} \frac{\gamma^{\prime \prime}\left(t_{2}\right)^{1 / 2} \gamma^{\prime}\left(t_{2}\right)^{\frac{n-2}{2}}}{\left|\xi_{n}\right|^{1 / 2} \gamma^{\prime \prime}\left(t_{1}\right)^{1 / 2}} \\
& \leq \frac{C(1+|\alpha|)}{\left|\xi^{\prime}\right|^{\frac{n-2}{2}}\left|\xi_{n}\right|^{1 / 2}}\left(\frac{\gamma^{\prime \prime}\left(t_{2}\right)}{\gamma^{\prime \prime}\left(t_{1}\right)}\right)^{1 / 2}\left|\frac{\xi^{\prime}}{\xi_{n}}\right|^{\frac{n-2}{2}} \\
& \quad=\frac{C(1+|\alpha|)}{\left|\xi_{n}\right|^{\frac{n-1}{2}}}\left(\frac{\gamma^{\prime \prime}\left(t_{2}\right)}{\gamma^{\prime \prime}\left(t_{1}\right)}\right)^{1 / 2} .
\end{aligned}
$$


Now

$$
\begin{aligned}
\gamma^{\prime \prime}\left(t_{2}\right) & \leq \frac{\gamma^{\prime}\left(t_{2}\right)}{t_{2}}\left|\log ^{\mu} \frac{1}{\gamma\left(t_{2}\right)}\right| \\
& \leq C \frac{\gamma^{\prime}\left(t_{1}\right)}{t_{1}} \frac{t_{1}}{t_{2}}\left|\log ^{\mu}\right| \xi_{n}|| \\
& \leq C \gamma^{\prime \prime}\left(t_{1}\right)\left|\log ^{\mu}\right| \xi_{n}||,
\end{aligned}
$$

which gives the upper bound of $\frac{C(1+|\alpha|)}{\left|\xi_{n}\right|^{\frac{n-1}{2}}}\left|\log ^{\mu / 2}\right| \xi_{n}||$ for the integral.

For the remaining part of the integral we need to deal with the case $n=3$ separately. The argument for $n=3$ follows that used for $\left[\frac{1}{\left|\xi^{\prime}\right|}, 1\right]$ in the case where there was no critical point in the interval. For $n \geq 4$ we also follow the argument used in that setting and integrate by parts repeatedly. This gives

$$
\begin{aligned}
& \frac{1}{\left|\xi^{\prime}\right|^{(n-2) / 2}}\left|\int_{t_{2}}^{1} e^{i\left(\xi_{n} \gamma(r)+\left|\xi^{\prime}\right| r\right)} \phi(r)\left(1-\phi_{1}\left(\frac{r\left|\xi^{\prime}\right|}{2}\right)\right) K(r)^{1 / 2+i \alpha} r^{(n-2) / 2} d r\right| \\
& \leq \frac{1}{\left|\xi^{\prime}\right|^{(n-2) / 2}}\left\{\frac{\gamma^{\prime \prime}\left(t_{2}\right)^{1 / 2} \gamma^{\prime}\left(t_{2}\right)^{(n-2) / 2}}{\left|\xi_{n} \gamma^{\prime}\left(t_{2}\right)+\right| \xi^{\prime}||}\right. \\
& +\frac{1}{\left|\xi_{n} \gamma^{\prime}\left(t_{2}\right)+\right| \xi^{\prime}||} \sum_{i=0}^{k-1}\left|\left[D_{r}^{i}\left(\phi(r)\left(1-\phi_{1}\left(\frac{r\left|\xi^{\prime}\right|}{2}\right)\right) K(r)^{1 / 2+i \alpha} r^{(n-2) / 2}\right)\right]\left(t_{2}\right)\right| \\
& \left.+\left|\int_{t_{2}}^{1} D_{r}^{k}\left[\phi(r)\left(1-\phi_{1}\left(\frac{r\left|\xi^{\prime}\right|}{2}\right)\right) K(r)^{1 / 2+i \alpha} r^{(n-2) / 2}\right] e^{i\left(\xi_{n} \gamma(r)+\left|\xi^{\prime}\right| r\right)} d r\right|\right\} \\
& =\mathcal{A}+\mathcal{B}+\mathcal{C} .
\end{aligned}
$$

Now $\mathcal{A} \leq C \frac{\gamma^{\prime}\left(t_{2}\right)^{\frac{n-1}{2}}\left|\log ^{\mu / 2} \frac{1}{\gamma\left(t_{2}\right)}\right|}{\left|\xi^{\prime}\right|^{n / 2} t_{2}^{1 / 2}} \leq C \frac{\left|\log ^{\mu / 2}\right| \xi_{n}||}{\left|\xi_{n}\right|^{\frac{n-1}{2}}}$. We use Lemma 5.3 to estimate the terms in the sum for $\mathcal{B}$. This gives

$$
\begin{aligned}
\mathcal{B} & \leq \frac{C(1+|\alpha|)^{N}}{\left|\xi^{\prime}\right|^{n / 2}} \sum_{i=1}^{k-1} \frac{1}{\left|\xi_{n}\right|^{i}} \gamma^{\prime}\left(t_{2}\right)^{\frac{n-1}{2}-i} t_{2}^{-\frac{1}{2}-i}\left|\log ^{\mu(i+1 / 2)} \frac{1}{\gamma\left(t_{2}\right)}\right| \\
& \leq \frac{C(1+|\alpha|)^{N}}{\left|\xi_{n}\right|^{\frac{n-1}{2}}}\left|\log ^{\mu(k-1 / 2)}\right| \xi_{n}|| .
\end{aligned}
$$


Finally Lemma 5.3 and Van der Corput's lemma give an upper bound for $\mathcal{C}$ :

$$
\mathcal{C} \leq \frac{C(1+|\alpha|)^{N}}{\left|\xi^{\prime}\right|^{\frac{n-2}{2}}\left|\xi_{n}\right|^{k}}\left|\xi^{\prime}\right|^{\frac{2 k-1}{2}}\left|\log ^{\mu(k+1)}\right| \xi_{n}|| \max _{\left[t_{2}, 1\right]} \gamma^{\prime}(t)^{\frac{n-1}{2}-k} .
$$

If we choose $k=\left[\frac{n}{2}\right]$ this gives us the desired bound.

Case 2: $\frac{1}{\left|\xi^{\prime}\right|}<\gamma^{-1}\left(\frac{1}{\left|\xi_{n}\right|}\right)$.

In this case we need to bound

$$
\frac{1}{\left|\xi^{\prime}\right|^{\frac{n-2}{2}}}\left|\int_{\gamma^{-1}\left(\frac{1}{\left|\xi_{n}\right|}\right)}^{1} e^{i\left(\xi_{n} \gamma(r)+r\left|\xi^{\prime}\right|\right)} \phi(r) K(r)^{1 / 2+i \alpha} r^{\frac{n-2}{2}} d r\right| .
$$

All the arguments are similar to those already given. This completes the proof of Proposition 5.1.

\section{References}

[1] J.-G. BAK, Restrictions of Fourier transforms to flat curves in $\mathbf{R}^{2}$, Illinois J. Math. 38(2) (1994), 327-346.

[2] J. Bourgain, On the restriction and multiplier problems in $\mathbf{R}^{3}$, in: "Geometric aspects of functional analysis" (1989-90), Lecture Notes in Math. 1469, Springer, Berlin, 1991, pp. 179-191.

[3] J. Bourgain, Some new estimates on oscillatory integrals, in: "Essays on Fourier analysis in honor of Elias M. Stein" (Princeton, NJ, 1991), Princeton Math. Ser. 42, Princeton Univ. Press, Princeton, NJ, 1995, pp. 83-112.

[4] L. Brandolini, A. Iosevich and G. Travaglini, Spherical means and the restriction phenomenon, J. Fourier Anal. Appl. 7(4) (2001), 359-372.

[5] J. Bruna, A. Nagel and S. Wainger, Convex hypersurfaces and Fourier transforms, Ann. of Math. (2) 127(2) (1988), 333-365.

[6] M. Cowling, S. Disney, G. Mauceri and D. Müller, Damping oscillatory integrals, Invent. Math. 101(2) (1990), 237-260.

[7] S. W. Drury, Degenerate curves and harmonic analysis, Math. Proc. Cambridge Philos. Soc. 108(1) (1990), 89-96.

[8] J. Ginibre And G. Velo, Scattering theory in the energy space for a class of nonlinear Schrödinger equations, J. Math. Pures Appl. (9) 64(4) (1985), 363-401.

[9] A. Greenleaf, Principal curvature and harmonic analysis, Indiana Univ. Math. J. 30(4) (1981), 519-537. 
[10] A. Iosevich, Fourier transform, $L^{2}$ restriction theorem, and scaling, Boll. Unione Mat. Ital. Sez. B Artic. Ric. Mat. (8) 2(2) (1999), $383-387$.

[11] A. Iosevich and G. Lu, Sharpness results and Knapp's homogeneity argument, Canad. Math. Bull. 43(1) (2000), 63-68.

[12] C. E. Kenig, G. Ponce And L. Vega, Oscillatory integrals and regularity of dispersive equations, Indiana Univ. Math. J. 40(1) (1991), 33-69.

[13] A. M. Li, U. Simon And G. S. Zhao, "Global affine differential geometry of hypersurfaces", de Gruyter Expositions in Mathematics 11, Walter de Gruyter \& Co., Berlin, 1993.

[14] E. Lutwak, Extended affine surface area, Adv. Math. 85(1) (1991), 39-68.

[15] G. Marletta, Curvilinear maximal functions, D. Phil thesis, University of Sussex (1995).

[16] A. Moyua, A. Vargas and L. Vega, Schrödinger maximal function and restriction properties of the Fourier transform, Internat. Math. Res. Notices 1996(16) (1996), 793-815.

[17] A. Moyua, A. Vargas and L. Vega, Restriction theorems and maximal operators related to oscillatory integrals in $\mathbf{R}^{3}$, Duke Math. J. 96(3) (1999), 547-574.

[18] H. Schulz, On the decay of the Fourier transform of measures on hypersurfaces, generated by radial functions, and related restriction theorems, unpublished (1990).

[19] P. SJőLIN, Fourier multipliers and estimates of the Fourier transform of measures carried by smooth curves in $R^{2}$, Studia Math. 51 (1974), 169-182.

[20] C. D. Sogge, A sharp restriction theorem for degenerate curves in $\mathbf{R}^{2}$, Amer. J. Math. 109(2) (1987), 223-228.

[21] C. D. Sogge And E. M. Stein, Averages of functions over hypersurfaces in $\mathbf{R}^{n}$, Invent. Math. 82(3) (1985), 543-556.

[22] E. M. Stein, "Harmonic analysis: real-variable methods, orthogonality, and oscillatory integrals", Princeton Mathematical Series 43, Monographs in Harmonic Analysis III, Princeton University Press, Princeton, NJ, 1993.

[23] T. Tao, A. Vargas and L. Vega, A bilinear approach to the restriction and Kakeya conjectures, J. Amer. Math. Soc. 11(4) (1998), 967-1000.

[24] T. TAO And A. Vargas, A bilinear approach to cone multipliers. I. Restriction estimates, Geom. Funct. Anal. 10(1) (2000), 185-215. 
[25] P. A. Tomas, A restriction theorem for the Fourier transform, Bull. Amer. Math. Soc. 81 (1975), 477-478.

[26] G. N. Watson, "A Treatise on the Theory of Bessel Functions", Cambridge University Press, Cambridge, 1922.

[27] T. WolfF, An improved bound for Kakeya type maximal functions, Rev. Mat. Iberoamericana 11(3) (1995), 651-674.

[28] A. Zygmund, On Fourier coefficients and transforms of functions of two variables, Studia Math. 50 (1974), 189-201.

Anthony Carbery:

Department of Mathematics and Statistics

University of Edinburgh

Edinburgh EH9 2BJ

U.K.

E-mail address: A.Carbery@ed.ac.uk

Sarah Ziesler:

Department of Mathematics and Computer Science

Dominican University

River Forest, IL 60305

U.S.A.

E-mail address: ziesler@math.uchicago.edu

E-mail address: ziessara@dom.edu

Primera versió rebuda el 6 de novembre de 2001, darrera versió rebuda el 3 d'abril de 2002. 